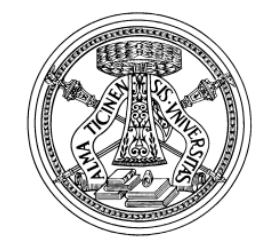

Quaderni di Dipartimento

\title{
Conjugate and Conditional Conjugate Bayesian Analysis of Discrete Graphical Models of Marginal Independence
}

\author{
Ioannis Ntzoufras
}

(Athens University of Economics and Business)

Claudia Tarantola

(Università di Pavia)

\author{
\# 178 (06-12) \\ Dipartimento di economia politica \\ e metodi quantitativi \\ Università degli studi di Pavia \\ Via San Felice, 5 \\ I-27100 Pavia
}

Giugno 2012 


\title{
Conjugate and Conditional Conjugate Bayesian Analysis of Discrete Graphical Models of Marginal Independence
}

\author{
Ioannis Ntzoufras* \\ Department of Statistics, Athens University of Economics and Business, Greece \\ Claudia Tarantola \\ Department of Economics and Business Sciences, University of Pavia, Italy
}

June 1, 2012

\begin{abstract}
We propose a conjugate and conditional conjugate Bayesian analysis of models of marginal independence with a bi-directed graph representation. We work with Markov equivalent directed acyclic graphs (DAGs) obtained using the same vertex set with the addition of some latent vertices when required. The DAG equivalent model is characterised by a minimal set of marginal and conditional probability parameters. This allows us to use compatible prior distributions based on products of Dirichlet distributions. For models with DAG representation on the same vertex set, the posterior distribution and the marginal likelihood is analytically available, while for the remaining ones a data augmentation scheme introducing additional latent variables is required. For the latter, we estimate the marginal likelihood using Chib's (1995) estimator. Additional implementation details including identifiability of such models is discussed. For all models, we also provide methodology for the computation of the posterior distributions of the marginal log-linear parameters based on a simple transformation of the simulated values of the probability parameters. We illustrate our method using a popular 4-way dataset.
\end{abstract}

Keywords: Bi-directed graph, Chib's marginal likelihood estimator, Contingency tables, Markov equivalent DAG, Monte Carlo computation.

*Address for correspondence: Ioannis Ntzoufras, Department of Statistics, Athens University of Economics and Business, Athens, Greece. E-mail: ntzoufras@aueb.gr 


\section{Introduction}

Graphical models of marginal independence were originally introduced by Cox and Wermuth (1993) for the analysis of multivariate Gaussian distributions, and subsequently extended to the discrete case by Drton and Richarson (2008a), Lupparelli (2006) and Lupparelli et al. (2009). They compose a family of multivariate distributions incorporating the marginal independences represented by a graph. The vertices in the graph correspond to a set of random variables, and the edges represent the pairwise associations between them. A missing edge from a pair of vertices indicates that the corresponding variables are marginally independent.

Despite the increasing interest in the literature for graphical models of marginal independence, Bayesian analysis has not been developed as much as traditional methods. For decomposable covariance graphical models, the problem has been successfully treated by Khare and Rajaratnam (2011). In the discrete case, some initial results regarding the analysis of three way contingency tables were presented by Ntzoufras and Tarantola (2012).

In this paper, we extend the work of Ntzoufras and Tarantola (2012) and we present a conjugate and conditional conjugate Bayesian analysis of discrete graphical models of marginal independencies. We exploit the connection between bi-directed graphs and directed acyclic graphs (DAGs). A bi-directed graph can be always represented in terms of a Markov equivalent DAG with the same set of vertices, or with some additional ones representing hidden or latent variables. The model is parameterised in terms of a set of marginal and conditional distributions, on which we assign conjugate priors based on products of Dirichlet distributions; see Heckerman et al. (1995). The marginal likelihood for models with DAG representation including latent variables is computed using the estimator of Chib (1995). Monte Carlo simulations are used to obtain the posterior distributions of the corresponding marginal log-linear parameters which have log-odds interpretation referring to marginal dependencies.

The plan of the paper is as follows. In Section 2, we introduce discrete graphical models of marginal independence, we establish the notation and we discuss their representation in terms of Markov equivalent DAGs. In Section 3, we present the probability parameterisation, the augmented likelihood factorisation, and the prior set-up. Section 4 is devoted to posterior inference, with particular emphasis on models with no direct DAG representation. The methodology is illustrated in Section 5 which presents the analysis of Coppen's (1966) dataset. Finally, in Section 6 , we conclude with a brief discussion. 


\section{Discrete Graphical Models of Marginal Independence}

\subsection{Bi-directed Graphs and Markov Properties}

In this section we briefly introduce graphical models of marginal independence, the related notation and terminology; for more details see, for example, in Drton and Richardson (2008a).

A bi-directed graph $G=(\mathcal{V}, E)$, is a graph with vertex set $\mathcal{V}$, and edge set $E$, such that $\left(v_{\imath}, v_{\jmath}\right) \in E$ if and only if $\left(v_{\jmath}, v_{\imath}\right) \in E$. We denote each bi-directed edge by $\left({\overrightarrow{v_{i}, v_{j}}}\right)=$ $\left\{\left(v_{i}, v_{j}\right),\left(v_{j}, v_{i}\right)\right\}$ and, following Richardson (2003), we represent it with a bi-directed arrow. An alternative representation, proposed by Cox and Wermuth (1993), is by undirected dashed edges.

The skeleton $\bar{G}$ of a bi-directed graph $G$ is the graph obtained by making all edges undirected; every triplet of vertices $\left(v_{\imath}, v_{\jmath}, v_{k}\right)$ in $\bar{G}$ with edges $\left(v_{\imath}, v_{\jmath}\right)$ and $\left(v_{\jmath}, v_{k}\right)$ is named $\vee$ configurations. A path connecting two vertices, $v_{0}$ and $v_{m}$, is a finite sequence of distinct vertices $v_{0}, \ldots v_{m}$ such that $\left(v_{i-1}, v_{\imath}\right), i=1, \ldots, m$, is an edge of the graph. A vertex set $\mathcal{C} \subseteq \mathcal{V}$ is connected if every two vertices $v_{\imath}$ and $v_{\jmath}$ are joined by a path in which every vertex is in $\mathcal{C}$. The vertex set $\mathcal{C} \subseteq \mathcal{V}$ induces a subgraph $G_{\mathcal{C}}$ obtained keeping only the edges having both end points in $\mathcal{C}$.

The graph is used to represent marginal independencies between a set of discrete random variables $X_{\mathcal{V}}=\left(X_{v}, v \in \mathcal{V}\right)$, each one taking values $i_{v} \in \mathcal{I}_{v}$; where $\mathcal{I}_{v}$ is the set of possible levels for variable $v$. The cross-tabulation of variables $X_{\mathcal{V}}$ produces a $|\mathcal{V}|$-way contingency table with cell frequencies $\boldsymbol{n}=(n(i), i \in \mathcal{I})$ where $\mathcal{I}=\mathbf{X}_{v \in \mathcal{V}} \mathcal{I}_{v}$. Similarly for any marginal $M \subseteq \mathcal{V}$, we denote with $X_{M}=\left(X_{v}, v \in M\right)$ the set of variables which produce the marginal table with frequencies $\boldsymbol{n}_{M}=\left(n_{M}\left(i_{M}\right), i_{M} \in \mathcal{I}_{M}\right)$ where $\mathcal{I}_{M}=\mathbf{X}_{v \in M} \mathcal{I}_{v}$.

The list of independencies implied by a bi-directed graph can be obtained using the following Markov properties: the pairwise Markov property (Cox and Wermuth, 1993) and the connected set Markov property (Richardson, 2003). The distribution of a random vector $X_{\mathcal{V}}$ satisfies the pairwise Markov property, if a missing edge in the graph indicates marginal independence between the corresponding variables. The distribution of a random vector $X_{\mathcal{V}}$ satisfies the connected set Markov property if for every disconnected set $\mathcal{D}$ the subvectors $X_{\mathcal{C}_{1}}, X_{\mathcal{C}_{2}}, \ldots, X_{\mathcal{C}_{r}}$, corresponding to its connected components $\mathcal{C}_{1}, \ldots, \mathcal{C}_{r}$, are mutually independent. For discrete variables the connected set Markov property implies the pairwise Markov property, whereas the converse is not generally true. Following Drton and Richardson (2008a), we define a discrete graphical model of marginal independence as the family of probability distributions for $X_{\mathcal{V}}$ that satisfy the connected set Markov property. 


\subsection{Representation in Terms of Markov Equivalent DAGs}

A bi-directed graph can always be represented via a Markov equivalent DAG with the same vertex set (D-decomposable graph) or with the introduction of some additional latent vertices; see Pearl and Wermuth (1994) and Drton and Richardson (2008b).

Given the skeleton $\bar{G}$ of the examined graph, one should assign arrows $v_{\imath} \longrightarrow v_{\jmath} \longleftarrow v_{k}$ to each $\vee$ configuration $\left(v_{\imath}, v_{\jmath}, v_{k}\right)$ in $\bar{G}$, constructing in this way the sink orientation of $G$. If no edge in the sink orientation is bi-directed the graph is D-decomposable. D-decomposable graphs do not include bi-directed 4-chain or the chordless 4-cycle sub-graphs. If the sink orientation contains bi-directed edges, a Markov equivalent DAG can be constructed substituting every bi-directed edge $v_{1} \longleftrightarrow v_{2}$ with the directed configuration $v_{1} \longleftarrow \ell \longrightarrow v_{2}$, where vertex $\ell$ represents a hidden or latent variable; see theorem 3 in Pearl and Wermuth (1994). We then obtain a new graphical structure, with $\ell$ being the parent vertex of the children $v_{1}$ and $v_{2}$. Finally, a Markov equivalent DAG is constructed via an acyclic orientation of the undirected edges present in the sink orientation of the graph.

Any DAG which is Markov equivalent to $G$ will be called augmented DAG of $G$. More precisely, let $\mathcal{L}$ be the set of hidden or latent vertices introduced in the graph to obtain a Markov equivalent DAG, and $X_{\mathcal{L}}=\left(X_{\ell}, \ell \in \mathcal{L}\right)$ be the corresponding vector of variables. The augmented DAG of $G$ is the graph representing the relation between the variables of $X_{\mathcal{A}}$, with $\mathcal{A}=\mathcal{V} \cup \mathcal{L}$. Naturally, if the bi-directed graph is D-decomposable $\mathcal{A}=\mathcal{V}$ since $\mathcal{L}=\emptyset$.

\section{$3 \quad$ Model Set-up}

In following, we work in terms of the augment DAG representation of the model, parameterising it via a minimal set of marginal and conditional probability parameters sufficient to obtain the joint distribution of interest.

\subsection{Probability Parameterisation and Augmented Likelihood Factorisation}

Given an augmented DAG representation $D$, the vector of joint probabilities $\boldsymbol{p}^{\mathcal{A}}(D)$ corresponding to the augmented set of variables $X_{\mathcal{A}}$ factorise as

$$
p^{\mathcal{A}}(i ; D)=\prod_{v \in \mathcal{A}} \pi_{v \mid p a(v ; D)}\left(i_{v} \mid i_{p a(v ; D)}\right)
$$

where $p a(v ; D)$ stands for the parents set of vertex $v$ in graph $D$, and $\pi_{v \mid U}\left(i_{v} \mid i_{U}\right)$ is the parameter for the conditional probability $P\left(X_{v}=i_{v} \mid X_{U}=i_{U}\right)$. The corresponding joint probabilities 
$\boldsymbol{p}(D)=\left(p(i ; D), i \in \mathcal{I}_{\mathcal{V}}\right)$ associated to the observable variables $X_{\mathcal{V}}$ are a function of $\boldsymbol{p}^{\mathcal{A}}(D)$, and are given by

$$
p(i ; D)=\sum_{i_{\ell} \in \mathcal{I}_{\mathcal{L}}} p^{\mathcal{A}}\left(i, i_{\ell} ; D\right)
$$

Since we focus on a specific augmented DAG, we simplify the notation by eliminating $D$ from $p a(v ; D), \pi_{v \mid p a(v ; D)}\left(i_{v} \mid i_{p a(v ; D)}\right), p^{\mathcal{A}}(i ; D)$ and $p(i ; D)$ appearing in (1) and (2). In the following, we work with a minimal set of probability parameters

$$
\boldsymbol{\pi}^{D}=\left(\boldsymbol{\pi}_{v \mid i_{p a(v)}} ; v \in \mathcal{A}, i_{p a(v)} \in \mathcal{I}_{p a(v)}\right) \text {, with } \boldsymbol{\pi}_{v \mid i_{p a(v)}}=\left(\pi_{v \mid p a(v)}\left(i_{v} \mid i_{p a(v)}\right) ; i_{v} \in \mathcal{I}_{v}\right) .
$$

This set refers to conditional and marginal probability parameters which are sufficient to reconstruct the joint probabilities $\boldsymbol{p}^{\mathcal{A}}$ under dependencies and independences induced by $D$. The augmented likelihood for a specific $D$, is given by

$$
f\left(\boldsymbol{n}^{\mathcal{A}} \mid \boldsymbol{\pi}^{D}\right)=\frac{\Gamma(N+1)}{\prod_{i \in \mathcal{I}_{\mathcal{A}}} \Gamma\left(n^{\mathcal{A}}(i)+1\right)} \prod_{v \in \mathcal{A}}\left\{\prod_{i_{c l(v)} \in \mathcal{I}_{c l(v)}} \pi_{v \mid p a(v)}\left(i_{v} \mid i_{p a(v)}\right)^{n^{\mathcal{A}}\left(i_{c l(v)}\right)}\right\}
$$

where $c l(v)=\{v\} \cup p a(v)$ and $\boldsymbol{n}^{\mathcal{A}}=\left(n^{\mathcal{A}}(i), i \in \mathcal{I}_{\mathcal{A}}\right)$ are the cell frequencies of the augmented contingency table for variables $\mathcal{A}$. If the bi-directed graph is D-decomposable, the DAG representation of $G$ does not includes any latent variables (i.e. $\mathcal{L}=\emptyset$ and $\mathcal{A}=\mathcal{V}$ ), hence the model likelihood is directly given by (3).

\subsection{Prior Distributions}

We use conjugate priors based on products of Dirichlet distributions; see Heckerman et al. (1995). We assign a Dirichlet prior on the probability parameters of each vertex conditionally on its parents resulting in the following prior set-up

$$
\begin{aligned}
f\left(\boldsymbol{\pi}^{D}\right) & =\prod_{v \in \mathcal{A}} \prod_{i_{p a(v)} \in \mathcal{I}_{p a(v)}} f_{\mathcal{D} i}\left(\boldsymbol{\pi}_{v \mid i_{p a(v)}} ; \boldsymbol{\alpha}_{v \mid i_{p a(v)}}\right) \\
& \propto \prod_{v \in \mathcal{A}}\left\{\prod_{i_{c l(v)} \in \mathcal{I}_{c l(v)}} \pi_{v \mid p a(v)}\left(i_{v} \mid i_{p a(v)}\right)^{\alpha_{c l(v)}\left(i_{c l(v)}\right)-1}\right\},
\end{aligned}
$$

where $\boldsymbol{\alpha}_{v \mid i_{p a(v)}}=\left(\alpha_{c l(v)}\left(i_{c l(v)}\right) ; i_{v} \in \mathcal{I}_{v}\right)$ and $f_{\mathcal{D} i}(\boldsymbol{\pi} ; \boldsymbol{\alpha})$ is the Dirichlet density function with parameters $\boldsymbol{\alpha}$ evaluated at $\boldsymbol{\pi}$.

In order to make the prior distributions "compatible" across models, we assign a Dirichlet distribution on the vector of joint probabilities $\boldsymbol{p}$ for the saturated model of the observed table with parameters $\boldsymbol{\alpha}=(\alpha(i), i \in \mathcal{I})$. If the model is not D-decomposable, we use a similar Dirichlet distribution with parameters $\alpha^{\mathcal{A}}(i)$ (for all $i \in \mathcal{I}^{\mathcal{A}}$ ) for the vector of joint probabilities 
$\boldsymbol{p}^{\mathcal{A}}$ of the saturated model on the augmented contingency table, such that the prior on $\boldsymbol{p}$ is the same as the the one considered initially. Thus, we obtain compatibility by setting $\alpha(i)=$ $\sum_{i_{\ell} \in \mathcal{I}_{\mathcal{L}}} \alpha^{\mathcal{A}}\left(i, i_{\ell}\right)$. Under this approach, each component $\boldsymbol{\pi}_{v \mid i_{p a(v)}}$ of $\boldsymbol{\pi}^{D}$ will a-priori follow the Dirichlet distribution appearing in (4) with each parameter calculated as

$$
\alpha_{c l(v)}\left(i_{c l(v)}\right)=\sum_{i_{\mathcal{A} \backslash c l(v)} \in \mathcal{I}_{\mathcal{A} \backslash c l(v)}} \alpha^{\mathcal{A}}\left(i_{c l(v)}, i_{\mathcal{A} \backslash c l(v)}\right) .
$$

More details on compatible prior distributions can be found in Dawid and Lauritzen (2000), Roverato and Consonni (2004), and Consonni and Veronese (2008) .

When no prior information is available, a usual choice is to consider equal $\alpha(i)$ for all cells $i \in \mathcal{I}$. Common choices for $\alpha(i)$ are $1 / 2$ (Jeffreys prior), 1 (unit expected cell prior, UEC) and $1 /|\mathcal{I}|$ (Perks prior, 1947); see Dellaportas and Forster (1999) for additional details. The choice of this prior parameter value is of prominent importance for the model comparison due to the well known sensitivity of the posterior model odds and the Bartlett-Lindley paradox (Lindley, 1957, Bartlett, 1957). In this paper, we present results for the previous prior choices. A detailed comparison of prior choices is presented in Table 2 of Ntzoufras and Tarantola (2012).

\section{Posterior Inference}

\subsection{Conditional Conjugate Analysis for non D-decomposable Models}

From (3) and (4), the posterior distribution of the parameters $\boldsymbol{\pi}^{D}$ given a set of augmented data $\boldsymbol{n}^{\mathcal{A}}$ is given by

$$
f\left(\boldsymbol{\pi}^{D} \mid \boldsymbol{n}^{\mathcal{A}}\right)=\prod_{v \in \mathcal{A}} \prod_{i_{p a(v)} \in \mathcal{I}_{p a(v)}} f_{\mathcal{D} i}\left(\boldsymbol{\pi}_{v \mid i_{p a(v)}} ; \widetilde{\boldsymbol{\alpha}}_{v \mid i_{p a(v)}}\right)
$$

where $\widetilde{\boldsymbol{\alpha}}_{v \mid i_{p a(v)}}=\left(\widetilde{\alpha}_{c l(v)}\left(i_{c l(v)}\right)=n_{c l(v)}^{\mathcal{A}}\left(i_{c l(v)}\right)+\alpha_{c l(v)}\left(i_{c l(v)}\right), i_{v} \in \mathcal{I}_{v}\right)$, for any given configuration $i_{p a(v)} \in \mathcal{I}_{p a(v)}$.

Moreover, the posterior distribution of the frequencies of the augmented table, $f\left(\boldsymbol{n}^{\mathcal{A}} \mid \boldsymbol{n}, \boldsymbol{\pi}^{D}\right)$, is given by

$$
\begin{aligned}
f\left(\boldsymbol{n}^{\mathcal{A}} \mid \boldsymbol{n}, \boldsymbol{\pi}^{D}\right) & \propto \prod_{i \in \mathcal{I}_{\mathcal{V}}} \prod_{i_{\ell} \in \mathcal{I}_{\mathcal{L}}} p^{\mathcal{A}}\left(i, i_{\ell}\right)^{n^{\mathcal{A}}\left(i, i_{\ell}\right)} I\left(n(i)=n_{\mathcal{V}}^{\mathcal{A}}(i)\right) \\
& =\prod_{i \in \mathcal{I}_{\mathcal{V}}} f_{m}\left(\boldsymbol{n}_{\mathcal{V}}^{\mathcal{A}}(i, \bullet) ; \varpi(i), n(i)\right)
\end{aligned}
$$

with

$$
\varpi(i)=\left(\varpi(i)=\frac{p^{\mathcal{A}}\left(i, i_{\ell}\right)}{\sum_{i_{L} \in \mathcal{I}_{\mathcal{L}}} p^{\mathcal{A}}\left(i, i_{L}\right)} ; i_{\ell} \in \mathcal{I}_{\mathcal{L}}\right),
$$


$\boldsymbol{n}_{\mathcal{V}}^{\mathcal{A}}(i, \bullet)$ being the $\left|\mathcal{I}_{\mathcal{L}}\right|$ dimensional vector of cell frequencies with elements $\left\{\left(n^{\mathcal{A}}\left(i, i_{\ell}\right)\right.\right.$ for all $i_{\ell} \in$ $\left.\mathcal{I}_{\mathcal{L}}\right\}$, and $n_{\mathcal{V}}^{\mathcal{A}}(i)=\sum_{i_{\ell} \in \mathcal{I}_{\mathcal{L}}} n^{\mathcal{A}}\left(i, i_{\ell}\right)$, for any given $i \in \mathcal{I}$. Moreover, we denote by $I(\cdot)$ the indicator function and by $f_{m}(\boldsymbol{n} ; \boldsymbol{\pi}, N)$ the probability function of the multinomial distribution with probability vector $\boldsymbol{\pi}$ and $N$ independent trials evaluated at $\boldsymbol{n}$.

In order to obtain a sample from the posterior distribution of $\boldsymbol{\pi}^{D}$ we consider the following Gibbs algorithm generating sequentially values from (5) and (6):

i) Generate the frequencies $\boldsymbol{n}^{\mathcal{A}}$ of an augmented table by randomly splitting every single cell $n(i)$ using $\boldsymbol{n}_{\mathcal{V}}^{\mathcal{A}}(i, \bullet) \sim \mathcal{M}$ ultinomial $(\varpi(i), n(i))$, for every $i \in \mathcal{I}$.

ii) For every $v \in \mathcal{A}$ and $i_{p a(v)} \in \mathcal{I}_{p a(v)}$ generate $\boldsymbol{\pi}_{v \mid i_{p a(v)}} \sim \operatorname{Dirichlet}\left(\widetilde{\boldsymbol{\alpha}}_{v \mid i_{p a(v)}}\right)$.

The second step of the algorithm should be applied only to parameters without any identifiability constraints; see Section 4.2 for more details. If one or more parameters in vector $\boldsymbol{\pi}_{v \mid i_{p a(v)}}$ are constrained, then the corresponding conditional Dirichlet distribution must be used in the conditional posterior distribution; for the properties of the Dirichlet distribution see, for example, in Table 2 of Frigyik et al. (2010).

Moreover, for both D-decomposable and non D-decomposable models, we can easily obtain a sample from the posterior distribution of the joint probabilities $\boldsymbol{p}$ by simply transforming each observation of the simulated sample of $\boldsymbol{\pi}^{D}$ using (1) and then summing over all levels of the latent variables as given by (2).

\subsection{Some Important Implementation Details}

The use of DAGs with latent variables to represent non D-decomposable models creates two problems which are common in latent variable modelling: non-identifiability and label switching.

Let us consider the identifiability problem first. In order to remain in the class of the Markov equivalent DAGs, the number of levels of the introduced latent variables should be such that the augmented DAG model has at least as many parameters as the one represented by the original bi-directed graph. Otherwise, the new model may impose additional undesirable dependencies or other constraints that are not implied by the bi-directed graph. Having this in mind, we suggest the following rules of thumb. First, we introduce latent variables with the least possible number of levels satisfying the restriction described above. At the second stage, we impose a number of constraints equal to the difference between the number of parameters of the models represented by the two Markov equivalent graphs (bi-directed graph and augmented DAG). We start imposing constraints from the probabilities of the latent variables and continuing, if 
necessary, to the probability parameters of the first level of each child in $D$ with at least one latent parent conditioned on the first levels of its parents. We propose to set the constrained parameters equal to the mean of the prior distribution we would like to impose on the parameters of the unconstrained version of the model. Thus, starting from the probabilities of the latent variables we set $\pi_{\ell}\left(i_{\ell}\right)=\alpha_{\ell}\left(i_{\ell}\right) / \sum_{i_{\ell} \in \mathcal{I}_{\ell}} \alpha_{\ell}\left(i_{\ell}\right)$ and

$$
\pi_{v \mid p a(v)}\left(i_{v}=1 \mid i_{p a(v)}=\{1\}^{|p a(v)|}\right)=\frac{\alpha_{c l(v)}\left(i_{v}=1, i_{p a(v)}=\{1\}^{|p a(v)|}\right)}{\sum_{i_{v} \in \mathcal{I}_{v}} \alpha_{c l(v)}\left(i_{v}=1, i_{p a(v)}=\{1\}^{|p a(v)|}\right)}
$$

for specific $v \in \mathcal{V}$ and its parents. For prior distributions with equal $\alpha(i)$ (as the prior set-ups we use here), these constraints simplify to $\pi_{\ell}\left(i_{\ell}\right)=1 /\left|\mathcal{I}_{\ell}\right|$ for $\ell \in \mathcal{L}$ and $\pi_{v \mid p a(v)}\left(i_{v}=1 \mid i_{p a(v)}=\right.$ $\left.\{1\}^{|p a(v)|}\right)=1 /\left|\mathcal{I}_{v}\right|$ for specific $v \in \mathcal{V}$ and its parents. This is indeed the parameterisation we have used in the illustration of Section 5 . Note that if one or more parameters in a vector $\boldsymbol{\pi}_{v \mid i_{p a}(v)}$ are constrained, then the prior distribution (4) should be modified using the corresponding conditional Dirichlet distributions.

An alternative is to implement the MCMC algorithm described in Section 4.1 on the unconstrained model. When informative priors are used, then constraints are indirectly imposed by them and the MCMC will produce results from the posterior distribution without any problem (returning the prior as posterior for unidentifiable variables). If flat, non-informative prior distributions are used, the MCMC output for the model parameters $\boldsymbol{\pi}^{D}$ will present a nonconvergence picture. Nevertheless, both joint probabilities $\boldsymbol{p}$ and marginal log-linear parameters $\lambda$ will converge to the appropriate target posterior distributions since they are both well defined. Therefore, a possible solution is to leave the MCMC run on the unconstrained model and focus on the interpretation of $\boldsymbol{p}$ and $\boldsymbol{\lambda}$.

Concerning the label switching problem, many approaches have been proposed in the literature such as imposing inequality constraints (see, e.g., Diebolt and Robert, 1994), re-labelling algorithms (see, e.g., Stephens, 2000), the random permutation sampler of Frühwirth-Schnatter (2001), and many others (see, e.g., Papastamoulis and Iliopoulos, 2010); see in Jasra et al. (2005) and Yao (2012) for a nice overview of the subject. Nevertheless, for the bi-directed 4chain graphs we have implemented, the MCMC was exploring only one of the alternative modes and therefore not causing any problems in the posterior inference. Even for bi-directed chordless 4-cycle graphs, where label switching is more intense due to the multiple permutations of the latent configurations, the joint probabilities and the log-linear parameters are not affected by this behaviour since they are identifiable with unimodal posterior distributions. Therefore, we have not pursued this issue further except for the computation of the marginal likelihood 
estimate where a correction for the label switching problem was implemented as we describe in Section 4.4.

\subsection{Marginal Log-Linear Parameters Estimation}

The marginal log-linear parameterisation for bi-directed graphs was proposed by Lupparelli (2006) and Lupparelli et. al. (2009) based on the class of marginal log-linear models of Bergsma and Rudas (2002). The marginal log-linear parameters can be obtained by

$$
\boldsymbol{\lambda}=\boldsymbol{C} \log (\boldsymbol{M} \operatorname{vec}(\boldsymbol{p}))
$$

where $\operatorname{vec}(\boldsymbol{p})$ is a vector of dimension $|\mathcal{I}|$ obtained by rearranging the elements $\boldsymbol{p}$ in a reverse lexicographical ordering of the corresponding variable levels with the level of the first variable changing first (or faster). The parameter vector $\boldsymbol{\lambda}$ satisfies sum-to-zero constraints, and $\boldsymbol{C}$ indicates the corresponding contrast matrix. Finally $\boldsymbol{M}$ is the marginalization matrix which specifies from which marginal we calculate each element of $\boldsymbol{\lambda}$. Details for the construction of $\boldsymbol{C}$ and $\boldsymbol{M}$ are available at the Appendix of Ntzoufras and Tarantola (2012).

In order to obtain a marginal log-linear parameterisation for a bi-directed graph $G$, the disconnected sets of the graph should be considered as marginals, with eventually the addition of the full set of variables (if the graph is connected). The marginal should be arranged according to a hierarchical ordering (see Bergsma and Rudas, 2002). Then zero constraints on specific marginal log-linear parameters are imposed; see Lupparelli et. al. (2009) for more details.

The marginal log-linear modelling set-up is expressed in terms of log-odds ratios referring to specific marginal tables. When an edge is absent from the bi-directed graph $G$, then the corresponding $\lambda$ parameters (i.e. the corresponding log-odds ratio) are constrained to zero. This parameterisation is useful in cases when information is available for specific marginal associations via odds ratios (i.e. marginal log-linear parameters) or when partial information (i.e. marginals) is available. Unfortunately equation (8) cannot be used to obtain a closed form expression for $\boldsymbol{p}$, hence iterative procedures are needed to obtain the likelihood of the model for each set of $\boldsymbol{\lambda}$ values; see Rudas and Bergsma (2004) and Lupparelli (2006).

Working directly on graphs with marginal log-linear parameterisation is complicated. First of all, no conjugate or conditional conjugate analysis is feasible. Moreover, the likelihood cannot be written directly in a closed form. Nevertheless, with the approach presented in this work, we can estimate the posterior distribution of $\boldsymbol{\lambda}$ in a straightforward manner using Monte Carlo samples from the posterior distribution of $\boldsymbol{\pi}^{D}$. Specifically, a sample from the posterior distribution of $\boldsymbol{\lambda}$ can be generated by the following iterative procedure. At each iteration $\mathrm{t}$ ( for $t=1, \ldots, T$ ): 
i) Generate a random value $\boldsymbol{\pi}^{D(t)}$ from the posterior distribution of $\boldsymbol{\pi}^{D}$.

ii) Calculate the full table of probabilities $\boldsymbol{p}^{(t)}$ from $\boldsymbol{\pi}^{D(t)}$.

iii) Obtain the vector of marginal log-linear parameters, $\boldsymbol{\lambda}^{(t)}$ from $\boldsymbol{p}^{(t)}$ via equation (8).

The generated values $\left(\boldsymbol{\lambda}^{(t)} ; t=1,2, \ldots, T\right)$ can be used to estimate summaries of the posterior distribution $f(\boldsymbol{\lambda} \mid G)$ or obtain plots fully describing this distribution. A major advantage of this approach is that all zero constraints on $\boldsymbol{\lambda}$ are automatically imposed by construction.

\subsection{Chib's Marginal Likelihood Estimator of Marginal Likelihood}

In this Section, we illustrate how Chib's (1995) estimator can be used to evaluate the marginal likelihood for non D-decomposable models. An estimate of the marginal likelihood is given by

$$
\widehat{f}(\boldsymbol{n} \mid D)=\frac{f\left(\boldsymbol{n} \mid \boldsymbol{\pi}^{* D}\right) f\left(\boldsymbol{\pi}^{* D}\right)}{f\left(\boldsymbol{\pi}^{* D} \mid \boldsymbol{n}\right)}
$$

where $\pi^{* D}$ should be a point of high posterior density in order to get reliable estimates. The posterior mode, the posterior median or the posterior mean can be appropriate points that can be used in (9).

The likelihood is given by the probability function of a multinomial distribution with joint probabilities $\boldsymbol{p}^{*}$ evaluated at the observed data $\boldsymbol{n}$

$$
\log f\left(\boldsymbol{n} \mid \boldsymbol{p}^{*}, D\right)=\log \Gamma\left(\sum_{i \in \mathcal{I}} n(i)+1\right)-\sum_{i \in \mathcal{I}} \log \Gamma(n(i)+1)+\sum_{i \in \mathcal{I}} n(i) \log p^{*}(i)
$$

where $\boldsymbol{p}^{*}$ is given by (2) after calculating (1) with $\boldsymbol{\pi}^{D}=\boldsymbol{\pi}^{* D}$.

The posterior ordinate $f\left(\boldsymbol{\pi}^{* D} \mid \boldsymbol{n}\right)$ is given by

$$
f\left(\boldsymbol{\pi}^{* D} \mid \boldsymbol{n}\right)=\mathrm{E}_{\boldsymbol{n} \mathcal{A} \mid \boldsymbol{n}}\left[\prod_{v \in \mathcal{A}} \prod_{i_{p a(v)} \in \mathcal{I}_{p a(v)}} f_{\mathcal{D} i}\left(\boldsymbol{\pi}_{v \mid i_{p a(v)}}^{*} ; \widetilde{\boldsymbol{\alpha}}_{v \mid i_{p a(v)}}\right)\right],
$$

where the expectations are taken with respect to the posterior distribution of the latent data $\boldsymbol{n}^{\mathcal{A}}$. The above equation results directly from the procedure described in Section 2.1.2 of Chib (1995) by further assuming independence between $\boldsymbol{\pi}_{v \mid i_{p a(v)}}$ given the augmented table $\boldsymbol{n}^{\mathcal{A}}$ for all $v \in \mathcal{A}$ and $i_{p a(v)} \in \mathcal{I}_{p a(v)}$ when $\boldsymbol{n}^{\mathcal{A}}$ is available. So $\widehat{f}\left(\boldsymbol{\pi}^{* D} \mid \boldsymbol{n}\right)$ is finally estimated via

$$
\widehat{f}\left(\boldsymbol{\pi}^{* D} \mid \boldsymbol{n}\right)=\frac{1}{T} \sum_{t=1}^{T}\left\{\prod_{v \in \mathcal{A}} \prod_{i_{p a(v)} \in \mathcal{I}_{p a(v)}}\left[f_{\mathcal{D} i}\left(\boldsymbol{\pi}_{v \mid i_{p a(v)}}^{*} ; \widetilde{\boldsymbol{\alpha}}_{v \mid i_{p a(v)}}^{(t)}\right)\right]\right\}
$$

where $\widetilde{\boldsymbol{\alpha}}_{v \mid i_{p a(v)}}^{(t)}=\left(\widetilde{\alpha}_{c l(v)}^{(t)}\left(i_{c l(v)}\right)=n_{c l(v)}^{\mathcal{A}(t)}\left(i_{c l(v)}\right)+\alpha_{c l(v)}\left(i_{c l(v)}\right), i_{v} \in \mathcal{I}_{v}\right)$, for any given configuration $i_{p a(v)} \in \mathcal{I}_{p a(v)}$. In the above expression, the Dirichlet densities must be replaced by the 
corresponding conditional Dirichlet when one or more components of $\boldsymbol{\pi}_{v \mid i_{p a(v)}}$ are constrained. Additional details for the 4-chain and chordless 4-cycle bi-directed graphs are provided in Section 5 and at the Appendix.

Due to the label switching problem, we adjust the original estimator by the correction originally proposed by Neal (1998) and further developed in more detail by Marin and Robert (2008). Moreover, the mode (or values close to it) is most suitable choice for $\boldsymbol{\pi}^{* D}$ that can be used in the Chib's estimator since the mean and the median will be away from points of high posterior density if the MCMC explores all local modes. In cases that the MCMC visits only one of the permutations of the labels of the latent variables, then using the posterior mean and median in Chib's estimator also results in good estimates of the marginal likelihood.

\section{Illustrative Example: Coppen's Dataset}

We consider a dataset presented by Coppen (1966) regarding the interrelation between symptoms manifested by 362 psychiatric patients; see Table 1 . The symptoms are: $A \equiv$ stability (1=extroverted, $2=$ introverted); $B$ validity (1=energetic, $2=$ psychasthenic); $C \equiv$ acute depression (1=yes, $2=$ no); $D \equiv$ solidity ( $1=$ hysteric, $2=$ rigid $)$.

Table 1: Coppen's (1966) dataset on symptoms of psychiatric patients

\begin{tabular}{ccccccc} 
& & \multicolumn{2}{c}{$C=1$} & & \multicolumn{2}{c}{$C=2$} \\
\cline { 3 - 4 } \cline { 6 - 7 }$B$ & $D$ & $A=1$ & $A=2$ & & $A=1$ & $A=2$ \\
\hline 1 & 1 & 15 & 23 & & 25 & 14 \\
& 2 & 9 & 14 & & 46 & 47 \\
2 & 1 & 30 & 22 & & 22 & 8 \\
& 2 & 32 & 16 & & 27 & 14 \\
\hline \multicolumn{2}{l}{ A: stability; B: validity; $C:$ acute depression; D: solidity. }
\end{tabular}

This dataset has been already analysed with different type of graphical models by Wermuth (1976), Lupparelli et al. (2009) and Roverato et al. (2012). In particular Lupparelli et al. (2009) applied discrete graphical models of marginal independence the graph in Figure 1 (a).

We present posterior results for the bi-directed 4-chain graph of Figure 1(a) implemented by Lupparelli et al. (2009) and the closest bi-directed chordless 4-cycle graph depicted in 1(b). Moreover, we present marginal likelihoods and posterior probabilities for all 4-vertex graphs. Posterior analysis for D-decomposable models can be implemented following the procedures described in Ntzoufras and Tarantola (2012). All the analysis was performed using three prior 
Figure 1: Bi-directed 4-chain and chordless 4-cycle graphs fitted in Coppen's data.

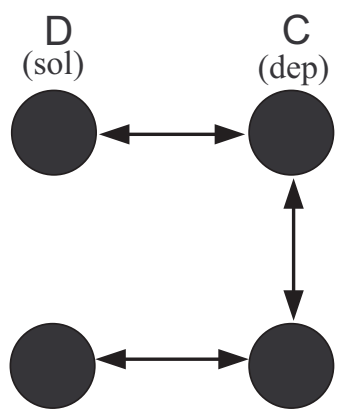

A

(sta) (val)

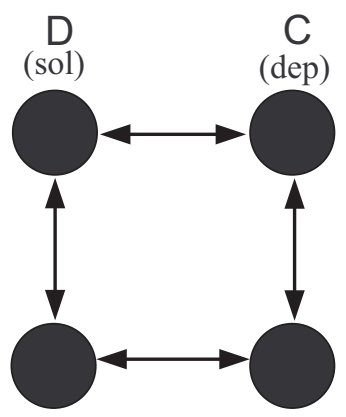

A

(sta)
B

(val)
(a) 4-chain graph
(b) 4-cycle graph
Notes. sta: stability; val: validity; dep: acute depression; sol: solidity.

choices for the saturated model of the observed table: the Perks prior (with $\alpha(i)=1 / 2^{4}$ ), the Jeffreys prior (with $\alpha(i)=1 / 2$ ) and the unit expected cells prior (with $\alpha(i)=1$ ). The priors of all other models have been designed to be compatible with these three baseline priors following the procedure described in Section 3.2. All results were obtained using R version 2.12.

Illustration on 4-chain $A B+B C+C D$ and chordless 4-cycle $A B+B C+C D+D A$

\section{bi-directed graphs.}

Here we present results for the bi-directed 4-chain graph $A B+B C+C D$ and the bi-directed chordless 4-cycle graph $A B+B C+C D+D A$. Both graphs have vertex set $\mathcal{V}=(A, B, C, D)$, the edge set of the first one is $E=\{(\overleftrightarrow{A, B}),(\overleftrightarrow{B, C}),(\overleftarrow{C, D})\}$, while for the second we consider the additional edge $(\overleftrightarrow{A, D})$. To obtain posterior summaries, we follow the general approach described in Sections 4.1-4.3, while the marginal likelihood estimator is obtained using the methodology described in Section 4.4. We introduce an additional latent variable $\ell$ between vertices $B$ and $C$ for the aforementioned bi-directed 4-chain graph and four latent variables, denoted by $\mathcal{L}=\left\{\ell_{1}, \ell_{2}, \ell_{3}, \ell_{4}\right\}$, for the bi-directed chordless 4-cycle graph. By this way, we obtain DAGs which are Markov equivalent to the original bi-directed graphs. Additional details are presented at the Appendix.

Figures 2, 3 and 4 present box-plots of the posterior distributions for the parameters $\pi^{D}$, the joint probabilities $\boldsymbol{p}$ of the observed four-way table, and the marginal log-linear parameters $\boldsymbol{\lambda}$ for the fitted model corresponding to the bi-directed 4-chain graph. In these box-plots, although 
we observe some variability in the posterior distributions of the augmented parameters $\boldsymbol{\pi}^{D}$, the differences of $\boldsymbol{p}$ and $\boldsymbol{\lambda}$ are minor. The corresponding boxplots for the bi-directed chordless 4 cycle are provided in Figures 5, 6 and 7. Differences in model parameters $\boldsymbol{\pi}^{D}$ between the three prior set-ups are more obvious now but still the joint probabilities and the marginal log-linear parameters are close.

Tables 2 and 3 present the batch mean estimators of the marginal log-likelihood along with the standard deviation of the marginal log-likelihood across 30 batches (i.e. MCMC sub-samples) of 1000 and 10000 iterations. The latter provides an estimate of the Monte Carlo error for the marginal log-likelihood estimate of equivalent size. All results are presented using three point estimates $\boldsymbol{\pi}^{* D}$ for the model parameters $\boldsymbol{\pi}^{D}$ : the mode, the median and the mean.

Table 2: Marginal log-likelihood estimates for bi-directed 4-chain graph $A B+B C+C D$ fitted on Coppen data under Perks, Jeffreys and unit expected cell priors using different point estimates (averages and standard deviations over 30 samples are reported).

\begin{tabular}{lcccc}
\hline Point & & \multicolumn{3}{c}{ Prior Set-up } \\
\cline { 3 - 5 } estimate & & Perks & Jeffreys & UEC \\
$\boldsymbol{\pi}^{* D}$ & Iterations & $\alpha(i)=1 / 2^{4}$ & $\alpha(i)=1 / 2$ & $\alpha(i)=1$ \\
\hline Mode & 1000 & $-64.63(0.598)$ & $-56.75(0.225)$ & $-56.68(0.131)$ \\
& 10000 & $-64.94(0.535)$ & $-56.68(0.074)$ & $-56.68(0.040)$ \\
Median & 1000 & $-64.88(0.361)$ & $-56.66(0.156)$ & $-56.69(0.125)$ \\
& 10000 & $-64.67(0.098)$ & $-56.7(0.044)$ & $-56.68(0.038)$ \\
Mean & 1000 & $-64.94(0.624)$ & $-56.64(0.175)$ & $-56.68(0.119)$ \\
& 10000 & $-64.64(0.169)$ & $-56.7(0.046)$ & $-56.68(0.039)$ \\
\hline
\end{tabular}

For the bi-directed 4-chain graph, we observe that for 1000 iteration the Monte-Carlo error is of acceptable size (between 0.12 and 0.63 ) while for 10000 iterations the error becomes really low for almost all cases presented in Table 2 (less than 0.1 for all point estimates and prior choices except for the Perks prior using the mode and mean as point estimates with standard deviations 0.53 and 0.17 , respectively).

For the bi-directed chordless 4-cycle graph, the Monte Carlo errors are much higher than the corresponding ones in the bi-directed 4-chain graph. This is due to the inclusion of four latent variables, which makes the MCMC slower in terms of convergence. Using the mode as point estimate in the Chib's estimator provides more reliable estimates with Monte Carlo errors $(1.97-2.63)$ for 1000 iterations and $(1.39-2.22)$ for 10000 iterations.

\section{Model Comparison and Evaluation}

Table 4 presents results for models with average posterior probability (over 30 MCMC samples) higher than 0.001 under the selected prior distributions. For all models we report the batch 
Figure 2: Boxplots of the posterior distribution of the model parameters $\boldsymbol{\pi}^{D}$ for the bi-directed 4-chain graph $A B+B C+C D$ fitted on the Coppen's data

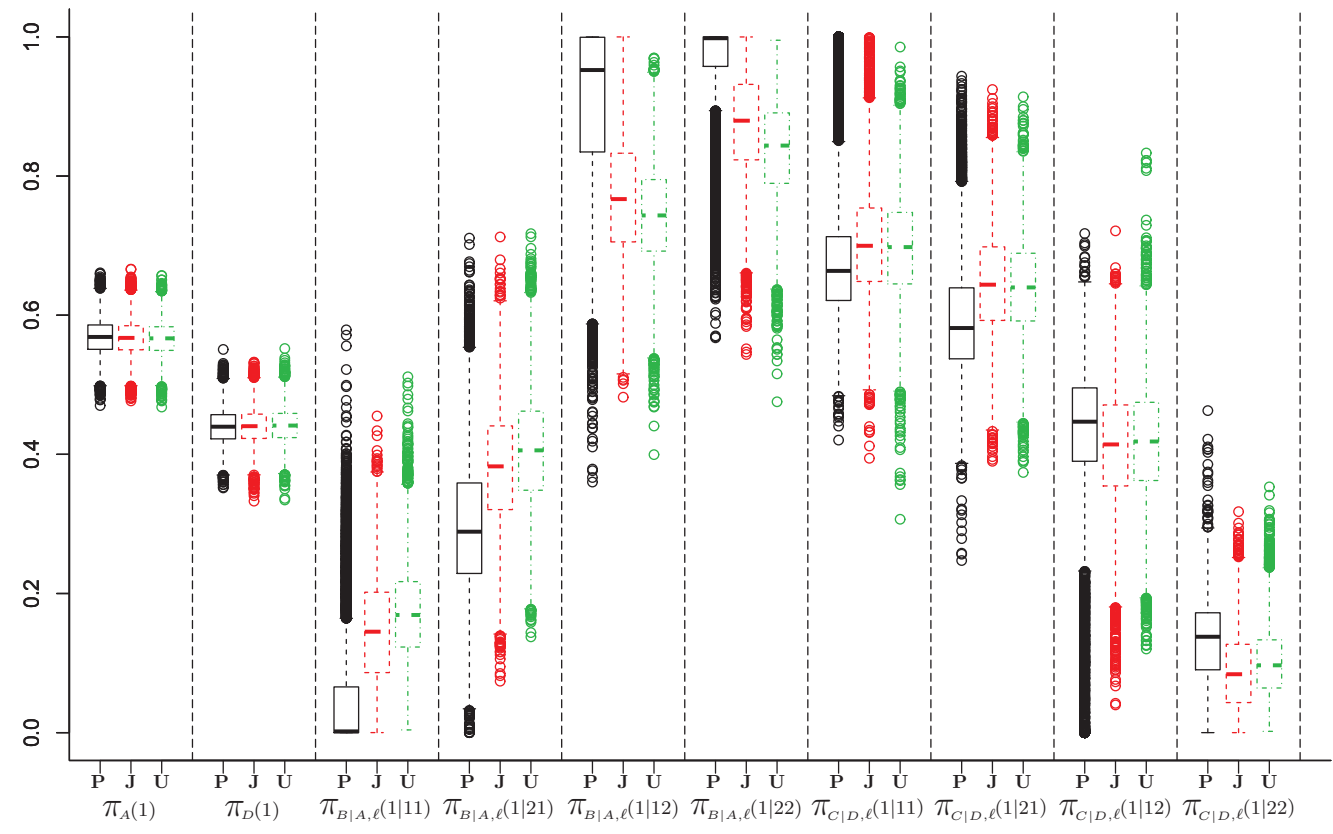

Prior distributions: $\mathrm{P}=$ Perks, $\mathrm{J}=$ Jeffreys, $\mathrm{U}=\mathrm{Unit}$ expected cell prior.

Figure 3: Boxplots of the posterior distribution of the joint probabilities $\boldsymbol{p}$ for the bi-directed 4-chain graph $A B+B C+C D$ fitted on the Coppen's data

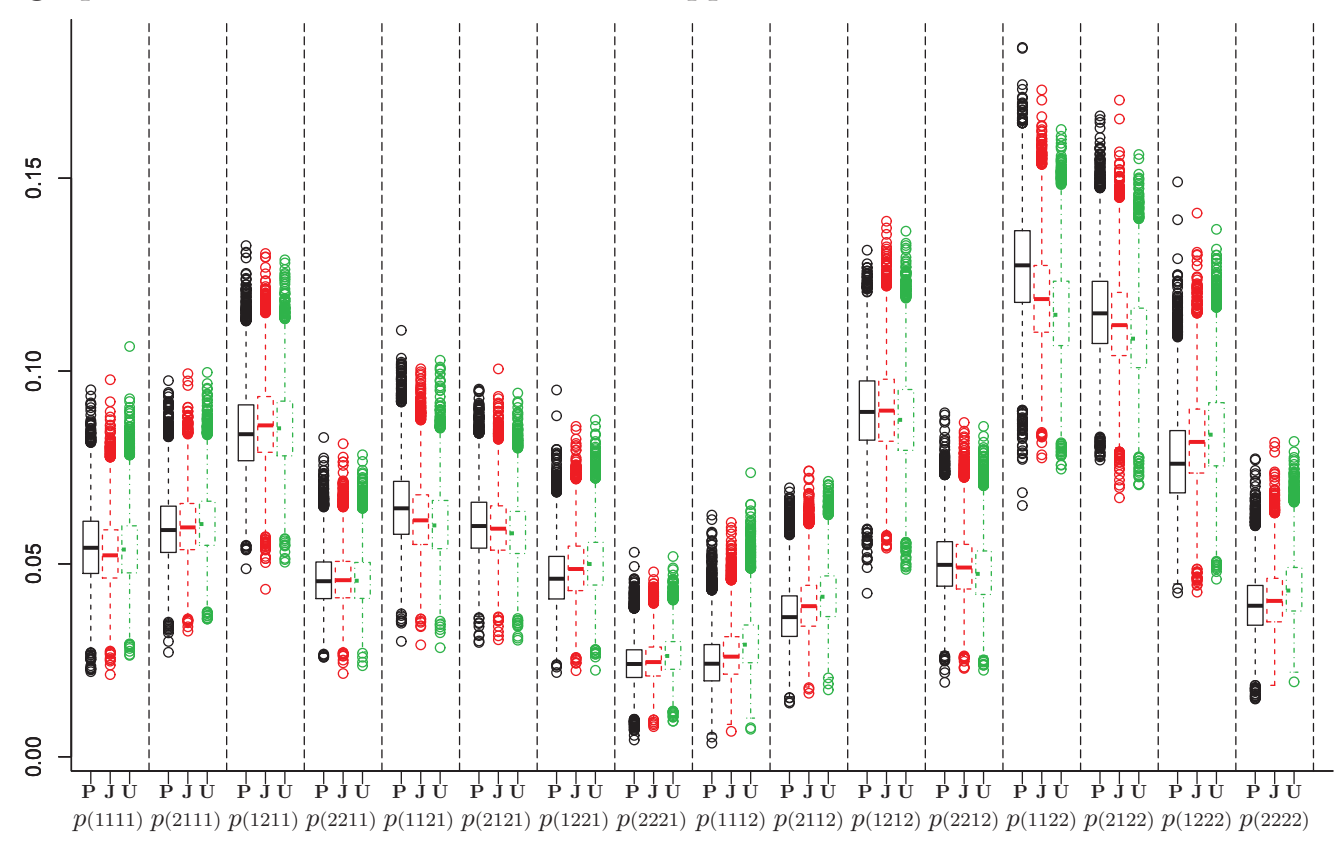

Prior distributions: $\mathrm{P}=$ Perks, $\mathrm{J}=$ Jeffreys, $\mathrm{U}=$ Unit expected cell prior. 
Figure 4: Boxplots of the posterior distribution of the marginal $\log$-linear parameters $\boldsymbol{\lambda}$ for the bi-directed 4-chain graph $A B+B C+C D$ fitted on the Coppen's data

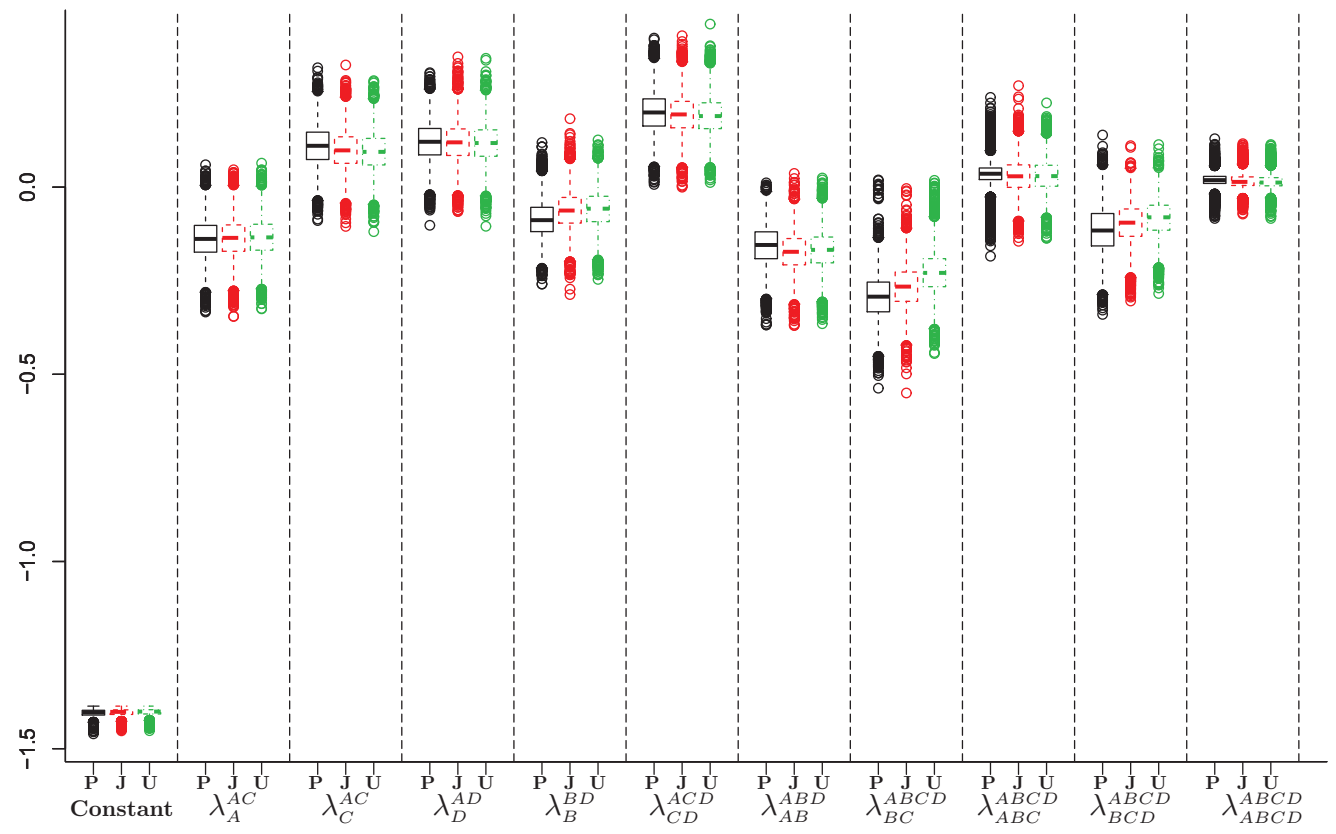

Prior distributions: $\mathrm{P}=$ Perks, $\mathrm{J}=$ Jeffreys, $\mathrm{U}=\mathrm{Unit}$ expected cell prior.

Notes for $\lambda$ 's: The constant term is estimated from the AC marginal; $\lambda_{T}^{M}$ denotes the parameter estimated for term $T$ from marginal $M$; all parameters refer to the second levels of each variable.

Figure 5: Boxplots of the posterior distribution of the model parameters $\pi^{D}$ for the bi-directed chordless 4-cycle graph $A B+B C+C D+D A$ fitted on the Coppen's data

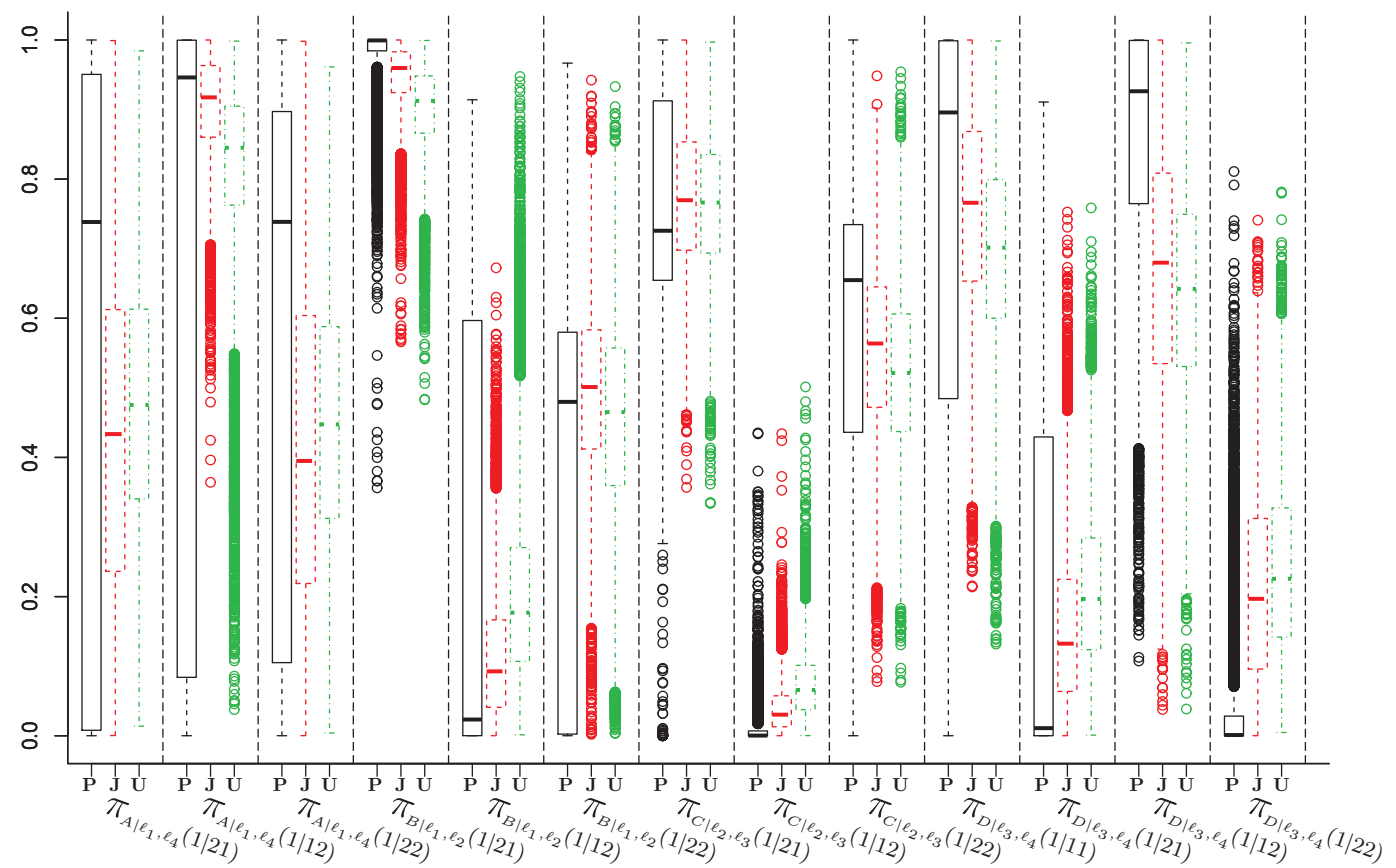

Prior distributions: $\mathrm{P}=$ Perks, $\mathrm{J}=$ Jeffreys, $\mathrm{U}=\mathrm{Unit}$ expected cell prior. 
Figure 6: Boxplots of the posterior distribution of the joint probabilities $\boldsymbol{p}$ for the bi-directed chordless 4-cycle graph $A B+B C+C D+D A$ fitted on the Coppen's data

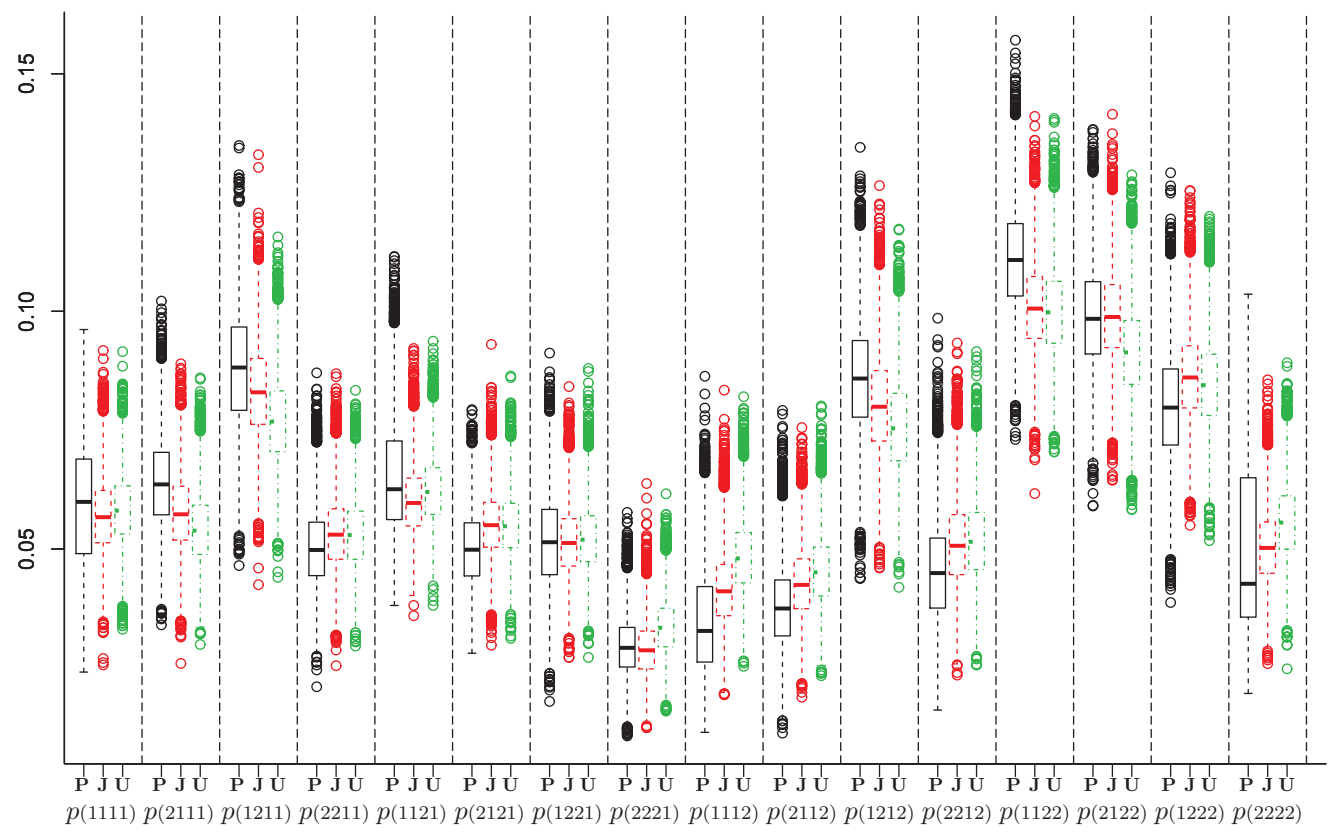

Prior distributions: $\mathrm{P}=$ Perks, $\mathrm{J}=$ Jeffreys, $\mathrm{U}=$ Unit expected cell prior.

Figure 7: Boxplots of the posterior distribution of the marginal log-linear parameters $\boldsymbol{\lambda}$ for the bi-directed chordless 4-cycle graph $A B+B C+C D+D A$ fitted on the Coppen's data

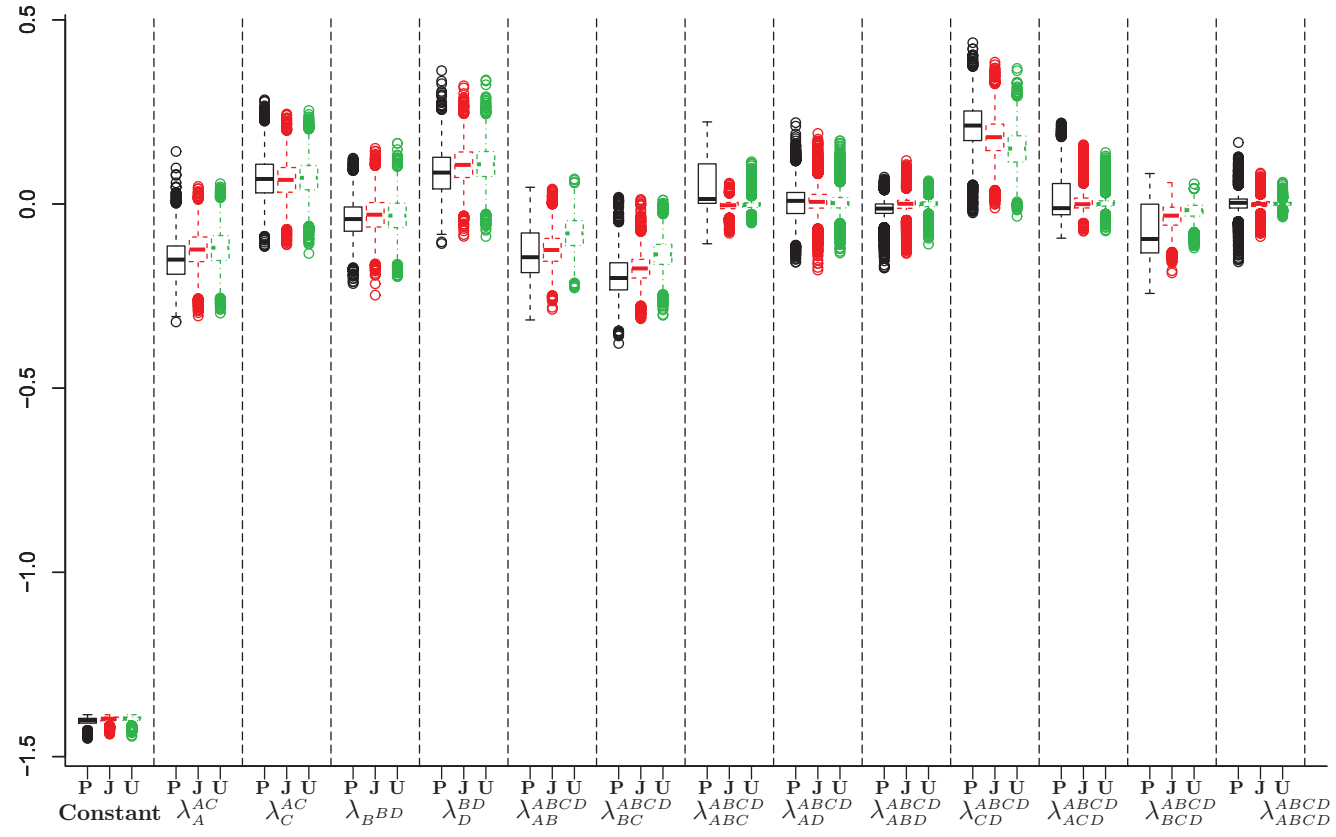

Prior distributions: $\mathrm{P}=$ Perks, $\mathrm{J}=$ Jeffreys, $\mathrm{U}=$ Unit expected cell prior.

Notes for $\lambda$ 's: The constant term is estimated from the AC marginal; $\lambda_{T}^{M}$ denotes the parameter estimated for term $T$ from marginal $\mathrm{M}$; all parameters refer to the second levels of each variable. 
Table 3: Marginal log-likelihood estimates for bi-directed chordless 4-cycle graph $A B+B C+$ $C D+D A$ fitted on Coppen data under Perks, Jeffreys and unit expected cell priors using different point estimates (averages and standard deviations over 30 samples are reported).

\begin{tabular}{lrccc}
\hline Point & & \multicolumn{3}{c}{ Prior Set-up } \\
\cline { 3 - 5 } estimate & & Perks & Jeffreys & UEC \\
$\boldsymbol{\pi}^{* D}$ & Iterations & $\alpha(i)=1 / 2^{4}$ & $\alpha(i)=1 / 2$ & $\alpha(i)=1$ \\
\hline Mode & 1000 & $-69.93(2.628)$ & $-67.65(2.460)$ & $-68.08(1.972)$ \\
& 10000 & $-66.76(2.056)$ & $-65.79(2.211)$ & $-66.78(1.393)$ \\
Median & 1000 & $-61.86(7.109)$ & $-62.40(1.999)$ & $-65.77(2.145)$ \\
& 10000 & $-62.36(3.244)$ & $-62.85(1.386)$ & $-66.54(1.240)$ \\
Mean & 1000 & $-52.59(8.895)$ & $-61.37(3.373)$ & $-65.14(3.723)$ \\
& 10000 & $-55.17(6.572)$ & $-62.25(2.220)$ & $-66.66(1.359)$ \\
\hline
\end{tabular}

mean estimate, its standard error and the standard deviation of the marginal log-likelihood and the corresponding posterior model probabilities over $30 \mathrm{MCMC}$ samples. In all simulations we have used 3000 iterations for the bi-directed 4-chain graphs and 10000 iterations for chordless 4-cycle graphs. Similar results are presented in Table 5 for the posterior inclusion probabilities of each edge of the graph.

Under all prior set-ups, the maximum a posteriori model (MAP) is the chain $A B+B C+C D$ with probabilities $0.67,0.91$ and 0.42 for Perks, Jeffreys and UEC prior, respectively. For the Perks prior, the relative difference between the MAP model and the second best (bi-directed chordless 4-cycle $\mathrm{AB}+\mathrm{BC}+\mathrm{CD}+\mathrm{DA}$ ) is smaller. The two models cannot be clearly distinguished (in terms of marginal likelihoods or posterior probabilities) when using 3000 or 10000 iterations for the estimation of the marginal likelihood. This is due to the large Monte Carlo error of the latter model ( \pm 2.03 for the log-marginal and \pm 0.26 for the posterior model probabilities). As a result we cannot safely identify differences between them even if the number of iterations for model $\mathrm{AB}+\mathrm{BC}+\mathrm{CD}+\mathrm{DA}$ is increased to 100000 iterations (marginal log-likelihood Monte Carlo error $\approx 1.24$ ).

For the rest of the prior distributions, all posterior model probabilities of the best models are accurately estimated. Higher levels of model uncertainty are observed for the UEC prior than the other two set-ups since 15 models have posterior model probability higher than $0.1 \%$ for the first in contrast to 5 and 7 models for the other two prior set-ups. For UEC prior, ABC+CD is supported as the second best model with estimated posterior probability 0.17 . This model ranked 5th in the Jeffreys prior and 23rd in Perks prior (with probabilities 0.007 and less than 0.001 , respectively). Model $\mathrm{A}+\mathrm{BC}+\mathrm{CD}$ is ranked high in all prior set-ups: 3rd, 2nd and 3rd for Perks, Jeffreys and UEC priors, respectively with posterior probabilities 0.010, 0.044 and 0.138.

Finally, Table 5 presents the summary statistics of the inclusion probabilities of each edge giving a clearer picture of the edges representing important dependencies. According to this 
table, edges $\mathrm{AB}, \mathrm{BC}$ and $\mathrm{CD}$ should be included in the finally selected graph with posterior inclusion probabilities at least $0.73,1.00$ and 0.99, respectively. Edge AD is mildly supported only under the Perks prior (inclusion probability $0.31 \pm 0.05$ ) while edges $\mathrm{AC}$ and $\mathrm{BD}$ are only weakly supported with inclusion probabilities around 0.23 for the UEC prior. Hence, the bidirected 4-chain graph $\mathrm{AB}+\mathrm{BC}+\mathrm{CD}$ is indicated as the median probability model (Barbieri and Berger, 2004) in all three prior set-ups.

\section{Discussion and Final Comments}

In this article, we have presented a novel Bayesian approach for the analysis of discrete graphical models of marginal independence. We have exploited the connection between bi-directed graphs and Markov equivalent DAGs by expressing them as models of conditional association. In this way, it was feasible to apply the recursive factorisation of the joint probability distribution of DAGs, and use suitable conjugate prior distributions. Posterior distributions were either readily available for parameters of bi-directed graphs with direct DAG representation, or estimated using a Gibbs sampler obtained by a data augmentation scheme. Chib's estimator was used to calculate the marginal likelihood of models without a direct DAG representation. Moreover, specific details were provided for the 4-way case along with an illustration in a well known dataset.

It worth noting that, the Markov equivalent DAG representation is not unique for all graphs. In this work, we have considered only one of the possible Markov equivalent DAGs. Nevertheless, since the prior distributions are compatible across models, the posterior distributions and the marginal likelihoods will not depend on this choice; see Buntine (1991) and Heckerman et al. (1995).

Even though the methodology presented here is general and can be applied for models of any dimension, its applicability to high dimensional contingency tables may be problematic in practice. This is due to the elevated number of latent variables that should be included in the Markov equivalent DAG. Therefore, for high dimensional problems, a more efficient methodology may be required. An alternative approach to estimate posterior model probabilities, on which we are working on, is to consider an appropriate trans-dimensional MCMC algorithm, see Sisson (2005) with emphasis given in reversible jump MCMC; Green (1995).

Finally, another interesting direction that we are currently considering, is to work directly with the marginal log-linear parameterisation $\boldsymbol{\lambda}$ defined by (8). In this case, a conjugate analysis is not feasible, and a more complicated approach is necessary. In this direction, two alternative approaches are under investigation: (a) an MCMC based directly on simulating the parameters of each marginal association log-linear parameters following the approach proposed by Knuiman and Speed (1988) and Dellaportas and Forster (1999), and (b) a Metropolis-Hastings algorithm with proposals based on the probability parameterisation we have considered in this article. A possible disadvantage of the first approach is that in each iteration of the MCMC sampler we 
Table 4: Marginal log-likelihood and posterior probabilities (\% values) for best models (with estimated posterior probability $>0.001$ ) for the Coppen's data (the batch mean estimates, standard errors, and standard deviations over 30 samples are reported); 3000 and 10000 iterations were used for the 4-chain and the chordless 4-cycle bi-directed graphs respectively.

\begin{tabular}{|c|c|c|c|c|c|}
\hline \multirow[b]{4}{*}{ Rank } & \multicolumn{5}{|c|}{ Perks Prior $\alpha(i)=1 / 2^{4}$} \\
\hline & & \multirow{2}{*}{\multicolumn{2}{|c|}{$\begin{array}{c}\text { Marginal } \\
\text { log-likelihood }\end{array}$}} & \multirow{2}{*}{\multicolumn{2}{|c|}{$\begin{array}{c}\text { Posterior } \\
\text { Probability (\%) }\end{array}$}} \\
\hline & & & & & \\
\hline & Model & Mean (S.E.) & S.D. & Mean (S.E.) & S.D. \\
\hline 1 & $\mathrm{AB}+\mathrm{BC}+\mathrm{CD}($ chain $)$ & $-64.75(0.095)$ & 0.523 & $67.31(4.791)$ & 26.24 \\
\hline 2 & $\mathrm{AB}+\mathrm{BC}+\mathrm{CD}+\mathrm{DA}($ cycle $)$ & $-66.21(0.370)$ & 2.029 & $30.85(4.893)$ & 26.80 \\
\hline 3 & $\mathrm{~A}+\mathrm{BC}+\mathrm{CD}$ & $-68.97(0.000)$ & 0.000 & $1.04(0.113)$ & 0.62 \\
\hline 4 & $\mathrm{AD}+\mathrm{BC}+\mathrm{CD}$ (chain) & $-69.89(0.083)$ & 0.457 & $0.49(0.095)$ & 0.52 \\
\hline \multirow[t]{4}{*}{5} & $\mathrm{~A}+\mathrm{BCD}$ & $-71.10(0.000)$ & 0.000 & $0.12(0.013)$ & 0.07 \\
\hline & \multicolumn{5}{|c|}{ Jeffreys Prior $\alpha(i)=1 / 2$} \\
\hline & & \multirow{2}{*}{\multicolumn{2}{|c|}{$\begin{array}{c}\text { Marginal } \\
\text { log-likelihood }\end{array}$}} & \multirow{2}{*}{\multicolumn{2}{|c|}{$\begin{array}{c}\text { Posterior } \\
\text { Probability (\%) }\end{array}$}} \\
\hline & & & & & \\
\hline Rank & Model & Mean (S.E.) & S.D. & Mean (S.E.) & S.D. \\
\hline 1 & $\mathrm{AB}+\mathrm{BC}+\mathrm{CD}$ (chain) & $-56.74(0.030)$ & 0.165 & $91.06(0.269)$ & 1.47 \\
\hline 2 & $\mathrm{~A}+\mathrm{BC}+\mathrm{CD}$ & $-59.79(0.000)$ & 0.000 & $4.37(0.126)$ & 0.69 \\
\hline 3 & $\mathrm{~A}+\mathrm{BCD}$ & $-60.44(0.000)$ & 0.000 & $2.27(0.065)$ & 0.36 \\
\hline 4 & $\mathrm{AD}+\mathrm{BC}+\mathrm{CD}$ (chain) & $-61.55(0.040)$ & 0.219 & $0.77(0.044)$ & 0.24 \\
\hline 5 & $\mathrm{ABC}+\mathrm{CD}$ & $-61.61(0.000)$ & 0.000 & $0.70(0.020)$ & 0.11 \\
\hline 6 & $\mathrm{AB}+\mathrm{BCD}$ & $-62.64(0.000)$ & 0.000 & $0.25(0.007)$ & 0.04 \\
\hline \multirow[t]{4}{*}{7} & $\mathrm{AB}+\mathrm{BC}+\mathrm{D}$ & $-62.89(0.000)$ & 0.000 & $0.20(0.006)$ & 0.03 \\
\hline & \multicolumn{5}{|c|}{ Unit Expected Cell Prior $\alpha(i)=1$} \\
\hline & & \multirow{2}{*}{\multicolumn{2}{|c|}{$\begin{array}{c}\text { Marginal } \\
\text { log-likelihood }\end{array}$}} & \multirow{2}{*}{\multicolumn{2}{|c|}{$\begin{array}{c}\text { Posterior } \\
\text { Probability (\%) }\end{array}$}} \\
\hline & & & & & \\
\hline Rank & Model & Mean (S.E.) & S.D. & Mean (S.E.) & S.D. \\
\hline 1 & $\mathrm{AB}+\mathrm{BC}+\mathrm{CD}$ (chain) & $-56.68(0.012)$ & 0.068 & $42.57(0.303)$ & 1.66 \\
\hline 2 & $\mathrm{ABC}+\mathrm{CD}$ & $-57.59(0.000)$ & 0.000 & $17.14(0.089)$ & 0.48 \\
\hline 3 & $\mathrm{~A}+\mathrm{BC}+\mathrm{CD}$ & $-57.81(0.000)$ & 0.000 & $13.76(0.071)$ & 0.39 \\
\hline 4 & $\mathrm{~A}+\mathrm{BCD}$ & $-58.11(0.000)$ & 0.000 & $10.2(0.053)$ & 0.29 \\
\hline 5 & $\mathrm{AB}+\mathrm{BCD}$ & $-58.56(0.000)$ & 0.000 & $6.55(0.034)$ & 0.19 \\
\hline 6 & $\mathrm{ABC}+\mathrm{BCD}$ & $-59.24(0.000)$ & 0.000 & $3.31(0.017)$ & 0.09 \\
\hline 7 & $\mathrm{ABD}+\mathrm{BDC}$ & $-59.89(0.000)$ & 0.000 & $1.72(0.009)$ & 0.05 \\
\hline 8 & $\mathrm{ACB}+\mathrm{ACD}$ & $-60.5(0.000)$ & 0.000 & $0.94(0.005)$ & 0.03 \\
\hline 9 & $\mathrm{AC}+\mathrm{BC}+\mathrm{CD}$ & $-60.77(0.000)$ & 0.000 & $0.71(0.004)$ & 0.02 \\
\hline 10 & $\mathrm{ABCD}$ & $-60.8(0.000)$ & 0.000 & $0.69(0.004)$ & 0.02 \\
\hline 11 & $\mathrm{AD}+\mathrm{BC}+\mathrm{CD}($ chain $)$ & $-60.91(0.018)$ & 0.099 & $0.63(0.013)$ & 0.07 \\
\hline 12 & $\mathrm{AB}+\mathrm{BC}+\mathrm{D}$ & $-60.95(0.000)$ & 0.000 & $0.60(0.003)$ & 0.02 \\
\hline 13 & $\mathrm{AC}+\mathrm{BCD}$ & $-61.07(0.000)$ & 0.000 & $0.53(0.003)$ & 0.01 \\
\hline 14 & $\mathrm{ABC}+\mathrm{D}$ & $-61.86(0.000)$ & 0.000 & $0.24(0.001)$ & 0.01 \\
\hline 15 & $\mathrm{ACD}+\mathrm{BC}$ & $-62.34(0.000)$ & 0.000 & $0.15(0.001)$ & 0.00 \\
\hline
\end{tabular}


Table 5: Posterior inclusion probabilities (\% values) for each edge of the bi-directed 4-way graph for the Coppen's data (the batch mean estimates (standard errors) over 30 samples are reported; 3000 and 10000 iterations were used for the 4-chain and the chordless 4-cycle bi-directed graphs respectively).

\begin{tabular}{lrrr}
\hline & \multicolumn{3}{c}{ Prior Set-up } \\
\cline { 2 - 4 } Edge & $\alpha(i)=1 / 16$ & $\alpha(i)=1 / 2$ & $\alpha(i)=1$ \\
\cline { 2 - 4 } AB & $98.2(0.22)$ & $92.5(0.22)$ & $73.8(0.14)$ \\
AC & $0.0(0.00)$ & $0.9(0.03)$ & $23.8(0.12)$ \\
AD & $31.4(4.85)$ & $0.9(0.06)$ & $4.3(0.03)$ \\
BC & $100.0(0.00)$ & $100.0(0.00)$ & $100.0(0.00)$ \\
BD & $0.1(0.01)$ & $2.7(0.08)$ & $23.1(0.12)$ \\
CD & $99.8(0.02)$ & $99.7(0.01)$ & $99.0(0.01)$ \\
\hline
\end{tabular}

need to implement iterative methods to calculate the cell probabilities and thus the calculation of the model likelihood will reduce the efficiency of the algorithm. Finally, implementing RJMCMC algorithm for the selection of the graphical structure seems a natural conclusion of this approach.

\section{Acknowledgements}

This work was partially supported by MIUR, Rome, under project PRIN 2005132307, University of Pavia, and by the Basic research funding program of the Research Centre of the Athens University of Economics and Business.

\section{References}

[1] Bartlett, M. S. (1957). A comment on Lindley's statistical paradox. Biometrika, 44, 533-534.

[2] Bergsma, W. P. and Rudas, T. (2002). Marginal log-linear models for categorical data. Annals of Statistics, 30, 140-159.

[3] Buntine, W. (1991). Theory refinement on Bayesian networks. In D'Ambrosio, P.S.B. and Bonissone, P. (eds.), Proceedings of the Conference on Uncertainty in Artificial Intelligence, 52-60. Morgan Kaufmann.

[4] Chib, S. (1995). Marginal Likelihood From the Gibbs Output. Journal of the American Statistical Association, 90, 1313-1321.

[5] Cox, D. R. and Wermuth, N. (1993). Linear dependencies represented by chain graphs (with discussion). Statistical Science, 8, 204-218, 247-277. 
[6] Consonni, G. and Veronese, P.(2008). Compatibility of Prior Specifications Across Linear Models. Statistical Science, 23, 332-353.

[7] Coppen, A. (1966). The Mark-Nyman temperament scale: an English translation. British Journal of Medical Psychology, 39, 55-59.

[8] Dawid, A.P. and Lauritzen, S.L. (2000). Compatible prior distributions. In Bayesian Methods with Applications to Science Policy and Official Statistics. The sixth world meeting of the International Society for Bayesian Analysis (ed. E.I. George), 109-118. http://www. stat.cmu.edu/ISBA/index.html.

[9] Dellaportas, P. and Forster, J.J. (1999). Markov Chain Monte Carlo model determination for hierarchical and graphical log-linear models. Biometrika, 86, 615-633.

[10] Diebolt, J., Robert, C.P. (1994). Estimation of finite mixture distributions through Bayesian sampling. Journal of the Royal Statistical Society B, 56, 363-375.

[11] Drton, M. and Richardson, T. S. (2008a). Binary models for marginal independence. Journal of the Royal Statistical Society B, 70, 287-309.

[12] Drton, M. and Richardson, T. S. (2008b). Graphical methods for efficient likelihood inference in Gaussian covariance models. Journal of Machine Learning Research, 9, 893-914.

[13] Frigyik, B.A., Kapila, A. and Gupta M.R. (2010). Introduction to the Dirichlet Distribution and Related Processes. Technical Report, UWEETR-2010-0006, Department of Electrical Engineering, University of Washington.

[14] Frühwirth-Schnatter, S. (2001). Markov chain Monte Carlo estimation of classical and dynamic switching and mixture models. Journal American Statistical Association, 96, 194-209.

[15] Green, P. J. (1995). Reversible jump Markov chain Monte Carlo computation and Bayesian model determination. Biometrika, 82, 711-732.

[16] Heckerman, D., Geiger, D. and Chickering, D. (1995). Learning Bayesian networks: the combination of knowledge and statistical data. Machine Learning, 20, 194-243.

[17] Jasra, A., Holmes, C.C. and Stephens, D.A. (2005). Markov Chain Monte Carlo Methods and the Label Switching Problem in Bayesian Mixture Modeling. Statistical Science, 20, $50-67$.

[18] Knuiman, M.W. and Speed, T. P. (1988). Incorporating prior information into the analysis of contingency tables. Biometrics, 44, 1061-1071.

[19] Khare, K. and Rajaratnam, B. (2011). Wishart distributions for decomposable covariance graph models. Annals of Statistics, 39, 514-555. 
[20] Lindley, D. V. (1957). A statistical paradox. Biometrika, 44, 187-192.

[21] Lupparelli, M. (2006). Graphical models of marginal independence for categorical variables. $\mathrm{Ph}$. D. thesis, University of Florence.

[22] Lupparelli M., Marchetti, G. M. and Bergsma, W. P. (2009). Parameterization and fitting of discrete bi-directed graph models. Scandinavian Journal of Statistics, 36, 559-576

[23] Marin, J.-M and Robert, C.P. (2008). Approximating the marginal likelihood in mixture models. Bulletin of the Indian Chapter of ISBA V(1), 2-7; also available as arXiv0804.2414 at http://arxiv.org/abs/0804.2414 .

[24] Neal, R.M. (1998). Erroneous Results in "Marginal Likelihood from the Gibbs Output" (with associated comments written 16 March 1999). Technical report, Department of Statistics and Department of Computer Science, University of Toronto, Canada; available at http://www.cs.toronto. edu/ radford/chib-letter.html.

[25] Ntzoufras, I. and Tarantola, C. (2012). Bayesian Analysis of Graphical Models of Marginal Independence for Three Way Contingency Tables, Technical report, Department of Political Economy and Quantitative Methods, University of Pavia.

[26] Papastamoulis, P., Iliopoulos, G. (2010). An artificial allocations based solution to the label switching problem in Bayesian analysis of mixtures of distributions. Journal of Computational and Graphical Statistics, 19, 313-331.

[27] Pearl, J. and Wermuth, N. (1994). When can association graphs admit a causal interpretation? In Models and data, artificial intelligence and statistics iv, Cheesman P. and Oldford,W., eds., Springer, New York, 205-214.

[28] Perks, W. (1947). Some observations on inverse probability including a new indifference rule. Journal of the institute of actuaries, 73, 285-334.

[29] Richardson, T. S. (2003). Markov property for acyclic directed mixed graphs. Scandinavian Journal of Statistics $\mathbf{3 0}, 145-157$.

[30] Roverato, A. and Consonni G. (2004) Compatible Prior Distributions for DAG models. Journal of the Royal Statistical Society B, 66, 47-61.

[31] Roverato, A., Lupparelli, M. and La Rocca, L. (2012). Log-mean linear models for binary data, arXiv:1109.6239v2

[32] Rudas, T. and Bergsma, W. P. (2004). On applications of marginal models for categorical data. Metron, LXII, 1-25.

[33] Sisson, S. A. (2005). Trans-dimensional Markov chains: A decade of progress and future perspectives. Journal of the Amarican Statistical association, 100, 1077-1089. 
[34] Stephens, M. (2000). Dealing with label switching in mixture models. Journal of the Royal Statistical Society B, 62, 795-809.

[35] Wermuth, N. (1976). Model search among multiplicative models. Biometrics, 32, 253-263.

[36] Yao, W. (2012). Model based labeling for mixture models. Statistics and Computing, 22, $337-347$. 


\section{Appendix}

\section{A Posterior Inference for a Bi-directed 4-chain Graph}

Here we provide specific details for the implementation of Bayesian inference for a bi-directed 4-chain graph. We consider the graph with vertex set $\mathcal{V}=\left\{e_{1}, c_{1}, c_{2}, e_{2}\right\}$ and edge set $E=\left\{\left(\overleftrightarrow{e_{1}, c_{1}}\right),\left(\overleftrightarrow{c_{1}, c_{2}}\right),\left(\overleftrightarrow{c_{2}, e_{2}}\right)\right\}$ represented in Figure $8($ a). This graph is Markov equivalent to a DAG with an additional latent variable $\ell$ added between $c_{1}$ and $c_{2}$, see Figure $8(\mathrm{~b})$. The joint probabilities (needed in the likelihood) for the original 4-way table are given by

$$
p(i)=\sum_{i_{\ell} \in \mathcal{I}_{\mathcal{L}}} p^{\mathcal{A}}\left(i, i_{\ell}\right)=\sum_{i_{\ell} \in \mathcal{I}_{\mathcal{L}}}\left\{\pi_{\ell}\left(i_{\ell}\right) \prod_{k=1}^{2} \pi_{e_{k}}\left(i_{e_{k}}\right) \pi_{c_{k} \mid e_{k}, \ell}\left(i_{c_{k}} \mid i_{e_{k}}, i_{\ell}\right)\right\}
$$

where $\mathcal{A}=\mathcal{V} \cup \mathcal{L}=\left\{e_{1}, c_{1}, c_{2}, e_{2}, \ell\right\}$ and $\mathcal{L}=\{\ell\}$.

Figure 8: Bi-directed and the Markov equivalent DAG representations for the bi-directed 4-chain graph

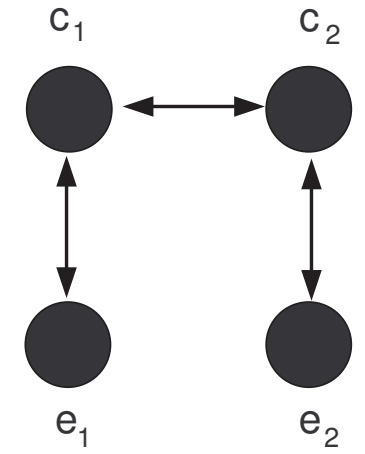

(a) Bi-directed graph

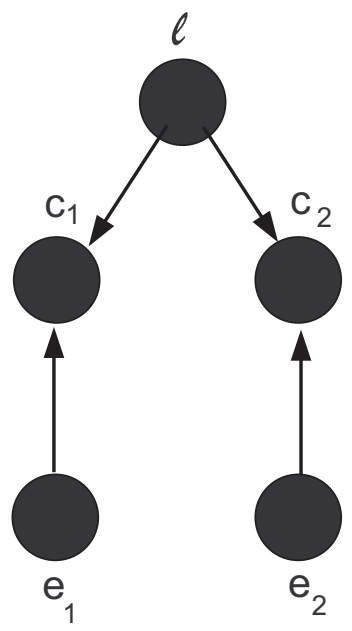

(b) DAG representation

The number of parameters in the above augmented model is equal to

$$
\mathrm{P}^{\mathrm{D}}=\sum_{k=1}^{2}\left(\left|\mathcal{I}_{e_{k}}\right|-1\right)+\sum_{k=1}^{2}\left(\left|\mathcal{I}_{c_{k}}\right|-1\right)\left|\mathcal{I}_{e_{k}}\right|\left|\mathcal{I}_{\ell}\right|+\left(\left|\mathcal{I}_{\ell}\right|-1\right)
$$

while the original model has

$$
\mathrm{P}^{\mathrm{G}}=\prod_{k=1}^{2}\left(\left|\mathcal{I}_{e_{k}}\right|\left|\mathcal{I}_{c_{k}}\right|+1\right)-\left(\prod_{k=1}^{2}\left|\mathcal{I}_{e_{k}}\right|\right)\left(\sum_{k=1}^{2}\left|\mathcal{I}_{c_{k}}\right|-1\right)-3
$$


parameters. The constraints are set using the approach described in Section 4.2. For the $2^{4}$ example implemented in Section 5 , we have $\mathrm{P}^{\mathrm{G}}=10$ and $\mathrm{P}^{\mathrm{D}}=11$ parameters when $\left|\mathcal{I}_{\ell}\right|=2$. Thus, only one constraint is needed. Here we have considered $\pi_{\ell}\left(i_{\ell}=1\right)=1 / 2$.

\section{A.1 Gibbs Sampling for a Bi-directed 4-chain Graph}

The Gibbs sampling described in Section 4.1 is implemented as follows:

1. Generate $n^{\mathcal{A}}\left(i, i_{\ell}\right) \sim \operatorname{Multinomial}(\widetilde{\boldsymbol{p}}(i), n)$ with $\widetilde{\boldsymbol{p}}(i)$ being a vector of length $\left|\mathcal{I}_{\ell}\right|$ and elements

$$
\widetilde{\boldsymbol{p}}\left(i, i_{\ell}\right)=\frac{p^{\mathcal{A}}\left(i, i_{\ell}\right)}{\sum_{i_{\ell}^{\prime} \in \mathcal{I}_{\mathcal{L}}} p^{\mathcal{A}}\left(i, i_{\ell}^{\prime}\right)}
$$

for $i_{\ell} \in \mathcal{I}_{\ell}$ and any $i \in \mathcal{I}$.

2. Generate $\pi_{e_{k}} \sim \operatorname{Dirichlet}\left(\boldsymbol{n}_{e_{k}}+\boldsymbol{\alpha}_{e_{k}}\right)$ for $k=1,2$.

3. Generate $\pi_{\ell} \sim \operatorname{Dirichlet}\left(\boldsymbol{n}_{\ell}^{\mathcal{A}}+\boldsymbol{\alpha}_{\ell}\right)$.

4. For $k=1,2, i_{e_{k}} \in \mathcal{I}_{e_{k}}$ and $i_{\ell} \in \mathcal{I}_{\ell}$, generate $\boldsymbol{\pi}_{c_{k} \mid i_{e_{k}}, i_{\ell}} \sim \operatorname{Dirichlet}\left(\boldsymbol{n}_{c_{k} \mid i_{e_{k}}, i_{\ell}}^{\mathcal{A}}+\boldsymbol{\alpha}_{c_{k} \mid i_{e_{k}}, i_{\ell}}\right)$.

The above MCMC implements the model with no constraints on $\boldsymbol{\pi}^{D}$. The constrained version of the model can be estimated in a similar way but in steps 3 and 4 the corresponding conditional Dirichlet distributions must be used instead. For the binary case presented in Section 5 , step 3 should be skipped since $\pi_{\ell}\left(i_{\ell}\right)=1 / 2$.

\section{A.2 Marginal Likelihood Computation for a Bi-directed 4-chain Graph}

For the estimation of the marginal likelihood, we use the Chib (1995) estimator as described in Section 4.4 using the output of the MCMC described in Appendix A.1 for the constrained version of the model. As $\boldsymbol{\pi}^{* D}$ we use three different points: the posterior mode, the posterior median and the posterior mean. The posterior mode is approximated via the MCMC output. Although using the MCMC is not the most efficient way to estimate the posterior mode, here the loss of the precision is not essential since the Chib's marginal likelihood estimator works well for any point of high posterior density.

The prior is simply the product of independent Dirichlet probability densities for each unconstrained component of $\boldsymbol{\pi}^{D}$ evaluated at $\boldsymbol{\pi}^{* D}$. The posterior ordinate $f\left(\boldsymbol{\pi}^{* D} \mid \boldsymbol{y}\right)$ is estimated from the Gibbs sampling output using the estimator 


$$
\begin{aligned}
\widehat{f}\left(\boldsymbol{\pi}^{* D} \mid \boldsymbol{y}\right) & =\prod_{k=1}^{2} f_{D i}\left(\boldsymbol{\pi}_{e_{k}}^{*} ; \boldsymbol{n}_{e_{k}}+\boldsymbol{\alpha}_{e_{k}}\right) \\
& \times \frac{1}{T} \sum_{t=1}^{T}\left\{f_{D i}\left(\boldsymbol{\pi}_{\ell}^{*} ; \boldsymbol{n}_{\ell}^{\mathcal{A}(t)}+\boldsymbol{\alpha}_{\ell}\right) \prod_{k=1}^{2} \prod_{i_{e_{k}} \in \mathcal{I}_{e_{k}}} \prod_{i_{\ell} \in \mathcal{I}_{v_{\ell}}} f_{D i}\left(\boldsymbol{\pi}_{c_{k} \mid e_{k}, \ell}^{*} ; \boldsymbol{n}_{c_{k} \mid e_{k}, \ell}^{\mathcal{A}(t)}+\boldsymbol{\alpha}_{c_{k} \mid e_{k}, \ell}\right)\right\},
\end{aligned}
$$

where $\boldsymbol{n}_{c_{k} \mid i_{e_{k}}, i_{\ell}}^{\mathcal{A}\left(i_{\ell}\right.}$ is a vector of frequency data with elements $n_{c_{k}, e_{k}, \ell}^{\mathcal{A}(t)}\left(i_{c_{k}}, i_{e_{k}}, i_{\ell}\right)$ for $i_{c_{k}} \in \mathcal{I}_{c_{k}}$ and given $i_{e_{k}}, i_{\ell}$. The superscript $(t)$ refers to the $t$-th iteration and $n^{\mathcal{A}(t)}(i)$ for $i \in \mathcal{I}_{\mathcal{A}}$ refers to the augmented 5 -way table after the introduction of the latent factor $\ell$ generated at the $t$-th iteration. Note that, in the equation above the densities must be replaced by the corresponding conditional Dirichlet densities if some parameters are constrained. For the binary case considered in Section 5 with $p\left(i_{\ell}=1\right)=1 / 2, f_{D i}\left(\boldsymbol{\pi}_{\ell}^{*} ; \boldsymbol{n}_{\ell}^{\mathcal{A}(t)}+\boldsymbol{\alpha}_{\ell}\right)$ must be eliminated from this expression. Finally, following Neal (1998) and Marin and Robert (2008), the marginal log-likelihood must be corrected by adding a factor equal to $\log \left(\left|\mathcal{I}_{\ell}\right|\right.$ !) which results to $\log 2=0.693$ for the case of a binary latent variable.

\section{B Posterior Inference for a Bi-directed Chordless 4-cycle Graph}

Here we provide the details for the implementation of the Bayesian inference for bidirected chordless 4 -cycle graphs with vertex set $\mathcal{V}=\left\{c_{1}, c_{2}, c_{3}, c_{4}\right\}$ and edge set $E=$ $\left\{\left(\overleftrightarrow{c_{1}, c_{2}}\right),\left(\overleftrightarrow{c_{2}, c_{3}}\right),\left(\overleftrightarrow{c_{3}, c_{4}}\right),\left(\overleftrightarrow{c_{4}, c_{1}}\right)\right\}$ represented in Figure $9($ a). This graph is Markov equivalent to a DAG with four additional latent variables $\mathcal{L}=\left\{\ell_{1}, \ell_{2}, \ell_{3}, \ell_{4}\right\}$ (see Figure $9(\mathrm{~b})$ ) with parameters $\pi_{\ell_{k}}$ and $\pi_{c_{k} \mid \ell_{k-1}, \ell_{k}}$ for $k=1,2,3,4$ and $\ell_{0}=\ell_{4}$. The joint probabilities for the original 4-way table are given by

$$
p(i)=\sum_{i_{L} \in \mathcal{I}_{\mathcal{L}}} p^{\mathcal{A}}\left(i, i_{\mathcal{L}}\right)=\sum_{i_{L} \in \mathcal{I}_{\mathcal{L}}} \prod_{k=1}^{4} \pi_{\ell_{k}}\left(i_{\ell_{k}}\right) \pi_{c_{k} \mid \ell_{k-1}, \ell_{k}}\left(i_{c_{k}} \mid i_{\ell_{k-1}}, i_{\ell_{k}}\right)
$$

where $\mathcal{A}=\mathcal{V} \cup \mathcal{L}=\left\{c_{1}, c_{2}, c_{3}, e_{4}, \ell_{1}, \ell_{2}, \ell_{3}, \ell_{4}\right\}$ and $i_{L}=\left\{i_{\ell_{1}}, i_{\ell_{2}}, i_{\ell_{3}}, i_{\ell_{4}}\right\}$.

\section{B.1 Gibbs Sampling for a Bi-directed Chordless 4-cycle Graph}

The MCMC scheme is similar to the one presented for the bi-directed 4-chain model and it can be summarized by the following steps:

1. Generate $n^{\mathcal{A}}\left(i, i_{\ell}\right) \sim \operatorname{Multinomial}(\widetilde{\boldsymbol{p}}(i), n)$ with $\widetilde{\boldsymbol{p}}(i)$ given by $(7)$. 
Figure 9: Bi-directed and the Markov equivalent DAG representations for the bi-directed chordless 4-cycle graph

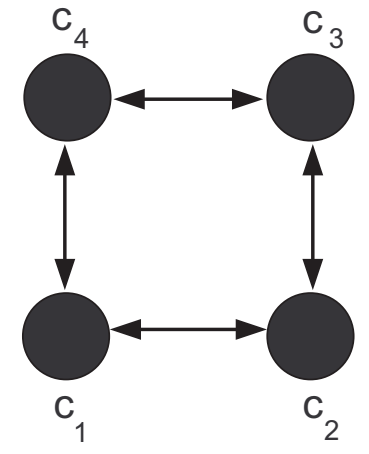

(a) Bi-directed graph

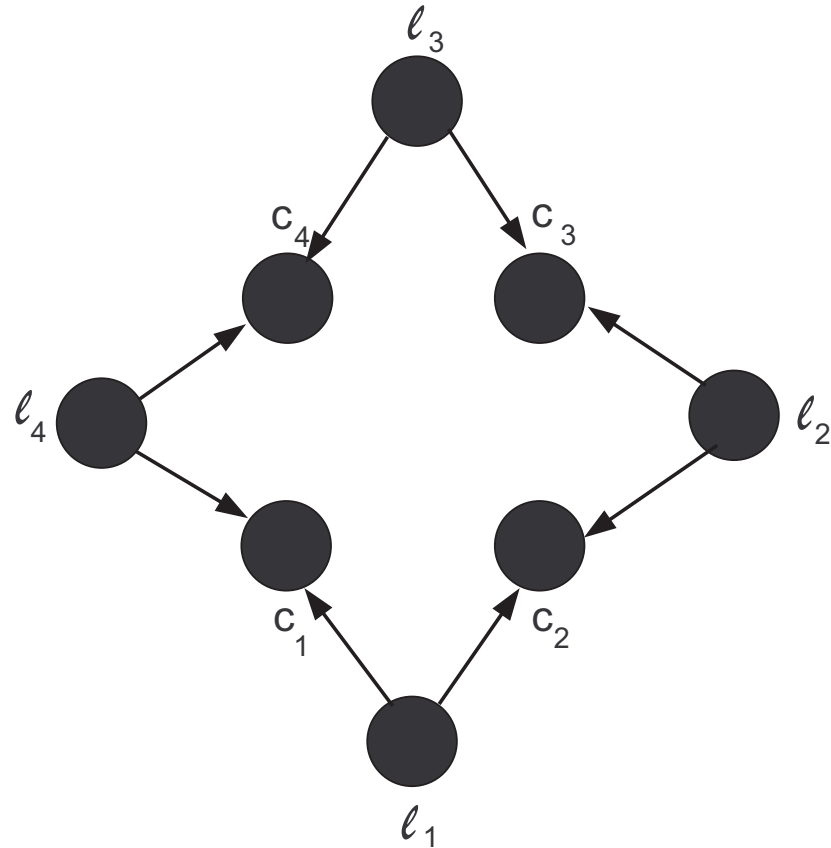

(b) DAG representation

2. For $c_{k} \in \mathcal{V}, i_{e_{k}} \in \mathcal{I}_{e_{k}}, i_{\ell} \in \mathcal{I}_{\ell}$, generate

$$
\boldsymbol{\pi}_{c_{k} \mid i_{\ell_{k-1}}, i_{\ell_{k}}} \sim \operatorname{Dirichlet}\left(\boldsymbol{n}_{c_{k} \mid i_{\ell_{k-1}}, i_{\ell_{k}}}^{\mathcal{A}}+\boldsymbol{\alpha}_{c_{k} \mid i_{\ell_{k-1}}, i_{\ell_{k}}}\right)
$$

with $\ell_{0}=\ell_{4}$.

3. For $\ell_{k} \in \mathcal{L}$, generate $\pi_{\ell_{k}} \sim \operatorname{Dirichlet}\left(\boldsymbol{n}_{\ell_{k}}^{\mathcal{A}}+\boldsymbol{\alpha}_{\ell_{k}}\right)$.

Similarly to Appendix A.1, the above sampler is for the unconstrained model. If the constrained version of the model is considered then steps 2 and 3 must be changed accordingly to accommodate these constraints.

The number of parameters in the bi-directed chordless 4-cycle graph presented in Figure 9(a) is given by

$$
\mathrm{P}^{\mathrm{G}}=\prod_{k=1}^{4}\left|\mathcal{I}_{c_{k}}\right|-\sum_{k=1}^{2}\left\{\prod_{j=0}^{1}\left|\mathcal{I}_{c_{k+2 j}}\right|\right\}-1
$$

while for the corresponding DAG, the number of parameters is given by

$$
\mathrm{P}^{\mathrm{D}}=\sum_{k=1}^{4}\left(\left|\mathcal{I}_{\ell_{k}}\right|-1\right)+\sum_{k=1}^{4}\left(\left|\mathcal{I}_{c_{k}}\right|-1\right)\left|\mathcal{I}_{\ell_{k-1}}\right|\left|\mathcal{I}_{\ell_{k}}\right| .
$$

For bi-directed chordless 4-cycle graphs with binary variables, we need to impose seven constraints since $\mathrm{P}^{\mathrm{G}}=2$ and $\mathrm{P}^{\mathrm{D}}=20$ for binary latent variables. In the illustration of 
Section 5 , we use $\pi_{\ell_{k}}\left(i_{\ell_{k}}\right)=1 / 2$ for all $i_{\ell_{k}} \in \mathcal{I}_{\ell_{k}}$ and $k=1,2,3,4$. Moreover, we set $\pi_{c_{k} \mid \ell_{k-1}, \ell_{k}}\left(i_{c_{k}} \mid i_{\ell_{k-1}}, i_{\ell_{k}}\right)=1 / 2$ for $k=1,2,3$.

\section{B.2 Marginal Likelihood Computation for Chordless Bi-directed 4-cycle Graphs}

The prior and the posterior ordinate involved in the estimation of the marginal likelihood using the estimator of Chib (1995) are now given by

$\log f\left(\boldsymbol{\pi}^{* D}\right)=\sum_{k=1}^{4}\left\{\log f_{D i}\left(\boldsymbol{\pi}_{\ell_{k}}^{*} ; \boldsymbol{\alpha}_{\ell_{k}}\right)+\sum_{i_{\ell_{k-1}} \in \mathcal{I}_{\ell_{k-1}}} \sum_{i_{\ell_{k}} \in \mathcal{I}_{\ell_{k}}} \log f_{D i}\left(\boldsymbol{\pi}_{c_{k} \mid i_{\ell_{k-1}}, i_{\ell_{k}}}^{*} ; \boldsymbol{\alpha}_{c_{k} \mid i_{\ell_{k-1}}, i_{\ell_{k}}}\right)\right\}$

and

$$
\begin{aligned}
\widehat{f}\left(\boldsymbol{\pi}^{* D} \mid \boldsymbol{y}\right)=\frac{1}{T} \sum_{t=1}^{T}\{ & \prod_{k=1}^{4} f_{D i}\left(\boldsymbol{\pi}_{\ell_{k}}^{*} ; \boldsymbol{\alpha}_{\ell_{k}}\right) \\
& \left.\times \prod_{k=1}^{4} \prod_{i_{\ell_{k-1}} \in \mathcal{I}_{\ell_{k-1}}} \prod_{i_{\ell_{k}} \in \mathcal{I}_{\ell_{\ell_{k}}}} f_{D i}\left(\boldsymbol{\pi}_{c_{k} \mid i_{\ell_{k-1}}, i_{\ell_{k}}}^{*} ; \boldsymbol{n}_{c_{k} \mid i_{\ell_{k-1}}, i_{\ell_{k}}}^{\mathcal{A}(t)}+\boldsymbol{\alpha}_{c_{k} \mid i_{\ell_{k-1}}, i_{\ell_{k}}}\right)\right\},
\end{aligned}
$$

respectively. Similarly to the implementation of the method for the 4-chain graph, in the expression above the densities must be substituted by the corresponding induced conditional Dirichlet densities if some parameters are constrained. Finally, to account for the label switching problem, the correction term that must be added in the marginal likelihood is equal to $\sum_{k=1}^{4} \log \left(\left|\mathcal{I}_{\ell_{k}}\right|\right.$ !) which results to $4 \log 2=2.77$ for the case of binary latent variables. 